\title{
Singularities of Maxwell's system in non-Hilbertian Sobolev spaces
}

\author{
Wided Chikouche And Serge Nicaise
}

\begin{abstract}
We study the regularity of the solution of the regularized electric Maxwell problem in a polygonal domain with data in $L^{p}(\Omega)^{2}$. Using a duality method, we prove a decomposition of the solution into a regular part in the nonHilbertian Sobolev space $W^{2, p}(\Omega)^{2}$ and an explicit singular one.
\end{abstract}

Mathematics Subject Classification (2000): 35A20 (primary); 35Q60, 78A25 (secondary).

\section{Introduction}

Let $\Omega$ be a bounded Lipschitz domain in $\mathbb{R}^{d}, d=2$ or 3 . The time harmonic Maxwell equations with perfect conductor boundary conditions can be written as

$$
\left\{\begin{array}{lll}
\operatorname{curl} \boldsymbol{E}-i k \boldsymbol{H}=0 & \text { and } \operatorname{curl} \boldsymbol{H}+i k \boldsymbol{E}=\boldsymbol{J} \text { in } \Omega, \\
\boldsymbol{E} \times \boldsymbol{n}=0 & \text { and } \boldsymbol{H} \cdot \boldsymbol{n}=0 & \text { on } \partial \Omega,
\end{array}\right.
$$

where $\boldsymbol{E}$ is the electric part and $\boldsymbol{H}$ the magnetic part of the electromagnetic field and $k \geq 0$ is the frequency of the electromagnetic wave. The right hand side $\boldsymbol{J}$ is the current density which in the absence of free electric charges is divergence free, namely

$$
\operatorname{div} \boldsymbol{J}=0 \quad \text { in } \Omega .
$$

If $k$ is different from zero, eliminating $\boldsymbol{H}$ by the relation $\boldsymbol{H}=\frac{1}{i k} \operatorname{curl} \boldsymbol{E}$, we deduce from (1.1) that the electric field $\boldsymbol{E}$ satisfies the second order system

$$
\begin{cases}\operatorname{curl} \operatorname{curl} \boldsymbol{E}-k^{2} \boldsymbol{E}=i k \boldsymbol{J} & \text { in } \Omega \\ \boldsymbol{E} \times \boldsymbol{n}=0 & \text { on } \partial \Omega\end{cases}
$$

When $\Omega$ is a non-convex polygonal or polyhedral domain, it is well know [5-8, $11,18]$ that the natural energy space for problem $(1.2)$ is not $H^{1}(\Omega)^{d}$ but only $H_{0}(\mathbf{c u r l} ; \Omega) \cap H(\operatorname{div} ; \Omega)$, the space of square integrable vector fields with square 
integrable rotational and divergence satisfying the tangential boundary conditions $\boldsymbol{E} \times \boldsymbol{n}=0$ on $\partial \Omega$. Moreover it was shown in [11] that if the right-hand side belongs to $L^{2}(\Omega)^{d}$, then this solution (in $H_{0}(\operatorname{curl} ; \Omega) \cap H(\operatorname{div} ; \Omega)$ ) is decomposed into the sum of a regular part in $H^{2}(\Omega)^{d}$ and a singular part, only determined by the singular functions of the Laplace operator with Dirichlet boundary conditions.

Regularity results with data in $L^{p}(\Omega)^{2}, p \neq 2$, seem to be useful for threedimensional numerical purposes $[4,20]$ and when one studies non linear equations related to Maxwell's system (like the MHD system, see [16]) by some kind of linearization or fixed point method. Hence it would be interesting to derive similar results than the ones cited above for data in $L^{p}(\Omega)^{2}$, with $p \neq 2$. In this paper, we start this analysis by considering the two-dimensional case. Namely using a duality method introduced by P. Grisvard in [15] for the Laplace operator, we obtain a decomposition (for the variational solution) into a regular part in $W^{2, p}(\Omega)^{2}$ and an explicit singular one. In a forthcoming paper, we will treat the three-dimensional case by combining the two-dimensional results obtained here with the theory of the sums of operators in Banach spaces, see for instance [2,9,12,13].

The paper is organized as follows. Section 2 is devoted to the setting of the problem, first we regularize the electric Maxwell problem by the addition of a grad div term to the operator curl curl in (1.2) which leads to a boundary value problem for the vectorial Laplace operator. Next we prove the existence and uniqueness of a variational solution in the space $H_{0}$ (curl) $\cap H$ (div) for data in $L^{p}(\Omega)^{2}$ with $p \geq \frac{4}{3}$. We conclude this section by a regularity result for the divergence of the variational solution.

In Section 3, we prove a priori estimates which allow to deduce that the vectorial Laplace operator is a semi-Fredholm operator in appropriate Sobolev spaces. This will be done by a local method and a change of variables which reduces our problem to a boundary value problem in an infinite strip studied in Subsection 3.1. There the solution is written explicitly by partial Fourier transform and thus estimated by applying Mikhlin's multiplier theorem.

In Section 4, in order to prove that the vectorial Laplace operator is Fredholm, we show that its kernel is reduced to $\{0\}$ and that the orthogonal of its range is finite dimensional. Finally we compute exactly the index of our problem by giving explicit functions which are not in the range of our operator. This will also lead to the desired decomposition into a regular part and a singular one.

\section{Setting of the problem}

Let $\Omega$ be a bounded polygonal domain of $\mathbb{R}^{2}$ with a Lipschitz boundary $\Gamma=$ $\cup_{j=1}^{N} \bar{\Gamma}_{j}$ with $\Gamma_{j}$ a linear segment, for all $j=1,2, \ldots, N$. Let us denote by $S_{j}$ the vertex of $\Omega$ defined by $S_{j}=\bar{\Gamma}_{j} \cap \bar{\Gamma}_{j+1}$ and by $\omega_{j}$ the opening of $\Omega$ at $S_{j}$.

Since we are interested in the regularity of the Maxwell system in $\Omega$ and since this regularity has a local character, without loss of generality we can assume that $\Omega$ is simply connected. 
As usual, we denote by $L^{p}(\Omega)$ and by $W^{k, p}(\Omega), k \in \mathbb{N}, p \in(1, \infty)$ the Lebesgue and Sobolev spaces. The usual norm of $W^{k, p}(\Omega)$ is denoted by $\|\cdot\|_{k, p, \Omega}$.

We consider the following electric Maxwell problem: given $\boldsymbol{J} \in L^{p}(\Omega)^{2}$, we look for a solution $\boldsymbol{E} \in W^{2, p}(\Omega)^{2}$ of

$$
\begin{cases}\operatorname{curl} \operatorname{curl} \boldsymbol{E}-k^{2} \boldsymbol{E}=\boldsymbol{J} & \text { in } \Omega, \\ \boldsymbol{E} \times \boldsymbol{n}=0 & \text { on } \Gamma,\end{cases}
$$

where $\boldsymbol{n}$ denotes the unit outer normal to $\Gamma$. The notations curl and curl distinguish between the scalar and vector curl operators:

$$
\operatorname{curl} \boldsymbol{u}=\frac{\partial u_{2}}{\partial x}-\frac{\partial u_{1}}{\partial y} \text { when } \boldsymbol{u}=\left(u_{1}, u_{2}\right)^{\top} \text { and } \operatorname{curl} \varphi=\left(\frac{\partial \varphi}{\partial y},-\frac{\partial \varphi}{\partial x}\right)^{\top},
$$

where we denote by $(x, y)$ the Cartesian coordinates in $\mathbb{R}^{2}$. We assume that

$$
\operatorname{div} \boldsymbol{J}=0 \quad \text { in } \Omega .
$$

With this hypothesis, it is clear that a solution of (2.1) satisfies $\operatorname{div} \boldsymbol{E}=0$ if $k \neq 0$, and thus, this is also a solution of

$$
\begin{cases}\operatorname{curl} \operatorname{curl} \boldsymbol{E}-\operatorname{grad} \operatorname{div} \boldsymbol{E}-k^{2} \boldsymbol{E}=\boldsymbol{J} & \text { in } \Omega, \\ \boldsymbol{E} \times \boldsymbol{n}=0 & \text { on } \Gamma, \\ \operatorname{div} \boldsymbol{E}=0 & \text { on } \Gamma .\end{cases}
$$

The divergence free condition $\operatorname{div} \boldsymbol{E}=0$ on $\Gamma$, is necessary to prove that conversely any solution $\boldsymbol{E}$ to (2.2) satisfies (2.1).

Instead of problem (2.2), we will consider along this paper the simpler Maxwell problem (2.3)-(2.5) below since its corner singularities has the same principal parts as problem (2.2) (see [11]),

$$
\begin{aligned}
& \text { curl } \operatorname{curl} \boldsymbol{E}-\operatorname{grad} \operatorname{div} \boldsymbol{E}=\boldsymbol{J} \text { in } \Omega, \\
& \boldsymbol{E} \times \boldsymbol{n}=0 \quad \text { on } \Gamma, \\
& \operatorname{div} \boldsymbol{E}=0 \quad \text { on } \Gamma \text {. }
\end{aligned}
$$

Since $-\Delta=\operatorname{curl} \operatorname{curl} \boldsymbol{E}-\operatorname{grad} \operatorname{div} \boldsymbol{E}$, equation (2.3) is equivalent to the vectorial Laplace equation

$$
-\Delta \boldsymbol{E}=\boldsymbol{J} \text { in } \Omega .
$$

\subsection{Variational formulation}

Let us introduce the spaces $H(\operatorname{curl} ; \Omega)$ and $H(\operatorname{div} ; \Omega)$

$$
\begin{gathered}
H(\operatorname{curl} ; \Omega)=\left\{\boldsymbol{u} \in L^{2}(\Omega)^{2} ; \operatorname{curl} \boldsymbol{u} \in L^{2}(\Omega)\right\}, \\
H(\operatorname{div} ; \Omega)=\left\{\boldsymbol{u} \in L^{2}(\Omega)^{2} ; \operatorname{div} \boldsymbol{u} \in L^{2}(\Omega)\right\} .
\end{gathered}
$$


The variational formulation of problem (2.3)-(2.5) consists in looking for $\boldsymbol{u} \in$ $X_{N}(\Omega)$ solution of

$$
\forall \boldsymbol{v} \in X_{N}(\Omega), \quad \int_{\Omega}(\operatorname{curl} \boldsymbol{u} \operatorname{curl} \boldsymbol{v}+\operatorname{div} \boldsymbol{u} \operatorname{div} \boldsymbol{v})=\int_{\Omega} \boldsymbol{J} \cdot \boldsymbol{v},
$$

where $X_{N}(\Omega)$ is the closed subspace of $H(\operatorname{curl} ; \Omega) \cap H(\operatorname{div} ; \Omega)$ defined as

$$
X_{N}(\Omega)=\{\boldsymbol{u} \in H(\operatorname{curl} ; \Omega) \cap H(\operatorname{div} ; \Omega) ; \boldsymbol{u} \times \boldsymbol{n}=0 \text { on } \Gamma\} .
$$

As $X_{N}(\Omega)$ is compactly embedded into $L^{2}(\Omega)^{2}$ [21] (see also [3, Th. 2.8]), the form

$$
a(\boldsymbol{u}, \boldsymbol{v})=\int_{\Omega}(\operatorname{curl} \boldsymbol{u} \operatorname{curl} \boldsymbol{v}+\operatorname{div} \boldsymbol{u} \operatorname{div} \boldsymbol{v}),
$$

is continuous and strongly coercive on $X_{N}(\Omega)$. Moreover as $X_{N}(\Omega)$ is embedded into $H^{1 / 2}(\Omega)^{2}[6,10,11]$, by the Sobolev embedding theorem, we deduce that $X_{N}(\Omega)$ is embedded into $L^{q}(\Omega)^{2}$, for all $1 \leq q \leq 4$ and therefore the linear form $v \rightarrow \int_{\Omega} \boldsymbol{J} \cdot \boldsymbol{v}$ is continuous on $X_{N}(\Omega)$ for all $p \geq \frac{4}{3}$. Applying the Lax-Milgram lemma we get the

Proposition 2.1. The variational problem (2.6) admits a unique solution $\boldsymbol{u} \in X_{N}(\Omega)$ for any $\boldsymbol{J} \in L^{p}(\Omega)^{2}$ such that $p \geq \frac{4}{3}$.

\subsection{The regularity of the divergence}

As for data in $\boldsymbol{J} \in L^{2}(\Omega)^{2}$ [11], we show that the divergence of the solution $\boldsymbol{u}$ of problem (2.6) with $p \geq \frac{4}{3}$ has a divergence with a better regularity.

Lemma 2.2. Let $\boldsymbol{u} \in X_{N}(\Omega)$ be the solution of (2.6) with $\boldsymbol{J} \in L^{p}(\Omega)^{2}$ such that $p \geq \frac{4}{3}$. Then

$$
\operatorname{div} \boldsymbol{u} \in W_{0}^{1, r}(\Omega) \text { with } r=\min \{p, 2\} .
$$

Proof. i) For $p \geq 2$, the result was proved in Theorem 1.2 of [11], where it was shown that $\operatorname{div} \boldsymbol{u}=q \in H_{0}^{1}(\Omega)$ is the unique solution of

$$
\int_{\Omega} \nabla q \cdot \nabla \varphi=-\int_{\Omega} \boldsymbol{J} \cdot \nabla \varphi, \forall \varphi \in H_{0}^{1}(\Omega) .
$$

ii) For $\frac{4}{3} \leq p<2$, we fix a sequence $\left(\boldsymbol{J}_{n}\right)_{n \in \mathbb{N}}$ of elements in $L^{2}(\Omega)^{2}$ such that

$$
\boldsymbol{J}_{n} \rightarrow \boldsymbol{J} \text { in } L^{p}(\Omega)^{2} \text { as } n \rightarrow \infty .
$$

We consider $\boldsymbol{u}_{n} \in X_{N}(\Omega)$ the solution of (2.6) with right hand side $\boldsymbol{J}_{n}$. Then from (2.8), we know that

$$
\boldsymbol{u}_{n} \rightarrow \boldsymbol{u} \text { in } X_{N}(\Omega) \text { as } n \rightarrow \infty .
$$


Applying the first item to $\boldsymbol{u}_{n}$, we deduce that $\operatorname{div} \boldsymbol{u}_{n}=q_{n} \in H_{0}^{1}(\Omega)$ is the unique solution of

$$
\int_{\Omega} \nabla q_{n} \cdot \nabla \varphi=-\int_{\Omega} \boldsymbol{J}_{n} \cdot \nabla \varphi, \forall \varphi \in H_{0}^{1}(\Omega)
$$

At this stage, for an arbitrary element $v \in L^{q}(\Omega)^{2}$, we use its Helmholtz decomposition

$$
v=\nabla r+\operatorname{curl} \psi
$$

where $r \in H_{0}^{1}(\Omega)$ is solution of

$$
\int_{\Omega} \nabla r \cdot \nabla \varphi=-\int_{\Omega} \boldsymbol{v} \cdot \nabla \varphi, \forall \varphi \in H_{0}^{1}(\Omega),
$$

and $\psi \in H^{1}(\Omega)$. This decomposition follows from [14, Theorem I.3.1] since $q>2$. Moreover from the elliptic regularity of the Laplace operator $r$ belongs to $W^{1, q}(\Omega)$ with the estimate

$$
\|r\|_{1, q, \Omega} \leq C\|\boldsymbol{v}\|_{0, q, \Omega},
$$

for some $C>0$. This is a consequence of [17, Theorems 8.1 and 8.2] since there is no singular exponents $\frac{k \pi}{\omega_{j}}$ between 0 and $1-\frac{2}{q}$.

Hence we may write

$$
\int_{\Omega} \nabla q_{n} \cdot \boldsymbol{v}=\int_{\Omega} \nabla q_{n} \cdot \nabla r=-\int_{\Omega} \boldsymbol{J}_{n} \cdot \nabla r,
$$

since by Green's formula $\int_{\Omega} \nabla q_{n} \cdot \operatorname{curl} \psi=-\int_{\Omega} q_{n} \cdot \operatorname{div} \operatorname{curl} \psi+\left\langle q_{n} ; \operatorname{curl} \psi \cdot \boldsymbol{n}\right\rangle=$ 0 (since $q_{n}=0$ on the boundary). By Hölder's inequality we obtain that

$$
\left|\int_{\Omega} \nabla q_{n} \cdot \boldsymbol{v}\right| \leq\left\|\boldsymbol{J}_{n}\right\|_{0, p, \Omega}\|\nabla r\|_{0, q, \Omega},
$$

and by (2.10), we get

$$
\left|\int_{\Omega} \nabla q_{n} \cdot \boldsymbol{v}\right| \leq C\left\|\boldsymbol{J}_{n}\right\|_{0, p, \Omega}\|\boldsymbol{v}\|_{0, q, \Omega} .
$$

Taking the supremum on $v \in L^{q}(\Omega)^{2}$, we have shown that

$$
\left\|\nabla q_{n}\right\|_{0, p, \Omega} \leq C\left\|\boldsymbol{J}_{n}\right\|_{0, p, \Omega} .
$$

This estimate and (2.8) and (2.9) allow to conclude that $\operatorname{div} \boldsymbol{u} \in W_{0}^{1, p}(\Omega)$. 
Remark 2.3. According to the previous theorem and [17, Theorems 8.1 and 8.2], for $p>2$, if $1-\frac{2}{p} \neq \frac{k \pi}{\omega_{j}}$ for all $j=1, \ldots, N, k \in \mathbb{N}^{\star}$, and if $\boldsymbol{u} \in X_{N}(\Omega)$ is the solution of (2.6) with $\boldsymbol{J} \in L^{p}(\Omega)^{2}$, then $\operatorname{div} \boldsymbol{u}$ satisfies

$$
\operatorname{div} \boldsymbol{u}=v_{R}+v_{S},
$$

where $v_{R} \in W_{0}^{1, p}(\Omega)$ and $v_{S} \in W_{0}^{1,2}(\Omega)$ is the singular part of $\operatorname{div} \boldsymbol{u}$ and is of the form

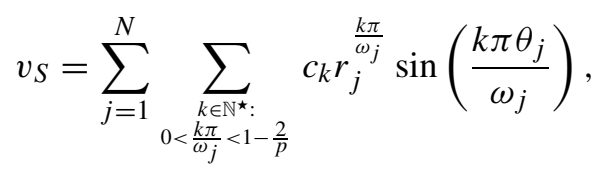

where $c_{k} \in \mathbb{R}$ and $\left(r_{j}, \theta_{j}\right)$ are polar coordinates centered at $S_{j}$ such that $\theta_{j}=0$ on $\Gamma_{j+1}$ and $\theta_{j}=\omega_{j}$ on $\Gamma_{j}$.

\section{The semi-Fredholm property}

In this section, we prove that our problem (2.3) -(2.5) has the semi-Fredholm property by proving an a priori estimate. For that purpose, we proceed locally (Subsection 3.2) in order to reduce our problem to a boundary value problem in an infinite strip, studied in Subsection 3.1. Such a strip has a smooth boundary, and we can use partial Fourier transform and Mikhlin's multiplier theorem to state the a priori estimate.

\subsection{A priori estimate in an infinite strip}

For a positive real number $\omega$, let $B$ be the infinite strip

$$
B=\mathbb{R} \times] 0, \omega\left[=\left\{(x, y) \in \mathbb{R}^{2} ; x \in \mathbb{R}, 0<y<\omega\right\} .\right.
$$

For a real number $r$ we consider the operator $L$ defined by

$$
L u=\partial_{x}^{2} u+\partial_{y}^{2} u+2 r \partial_{x} u+r^{2} u .
$$

Given $\boldsymbol{f} \in L^{p}(B)^{2}$, we look at $\boldsymbol{u}=\left(u_{1}, u_{2}\right)^{\top} \in W^{2, p}(B)^{2}$ a solution of

$$
\begin{cases}L u_{i}=f_{i} & \text { in } B, i=1,2, \\ u_{1} \cos \alpha+u_{2} \sin \alpha=0 & \text { on } F_{\alpha}, \alpha=0, \omega, \\ \sin \alpha \partial_{y} u_{1}-\cos \alpha \partial_{y} u_{2}=0 & \text { on } F_{\alpha}, \alpha=0, \omega\end{cases}
$$

where for $\alpha=0$ or $\omega$

$$
F_{\alpha}=\{(x, \alpha) ; x \in \mathbb{R}\}
$$


We are looking for a sufficient condition between $r$ and $\omega$ that guarantees the a priori estimate

$$
\|\boldsymbol{u}\|_{2, p, B} \leq c_{p}\|\boldsymbol{f}\|_{0, p, B}
$$

for some positive constant $c_{p}$.

For that purpose, we compute explicitly a solution $\boldsymbol{u}$ of (3.1) by applying partial Fourier transform in $x$ and then using Miklin's multiplier theorem.

\subsubsection{Explicit solution by partial Fourier transform}

After applying partial Fourier transform in $x$, problem (3.1) becomes

$$
\left\{\begin{array}{l}
\left.v_{i}^{\prime \prime}-\rho^{2} v_{i}=g_{i} \quad \text { in }\right] 0, \omega[, i=1,2, \\
v_{1}(\xi, 0)=v_{2}^{\prime}(\xi, 0)=0, \\
v_{1}(\xi, \omega) \cos \omega+v_{2}(\xi, \omega) \sin \omega=0, \\
\sin \omega v_{1}^{\prime}(\xi, \omega)-\cos \omega v_{2}^{\prime}(\xi, \omega)=0
\end{array}\right.
$$

for all $\xi \in \mathbb{R}$, where we have set $v_{i}=\widehat{u}_{i}, g_{i}=\widehat{f_{i}}$, and $\rho=\operatorname{sgn} \xi(-\xi+i r)$, where $\operatorname{sgn} \xi=1$ if $\xi \geq 0$ and -1 else. Any solution of the differential equation in (3.3) takes the form

$$
v_{j}(\xi, y)=\frac{1}{2 \rho} \int_{0}^{\omega} e^{\rho|y-z|} g_{j}(\xi, z) d z+a_{j} e^{\rho y}+a_{-j} e^{\rho(\omega-y)},
$$

for some $a_{j}, a_{-j} \in \mathbb{C}, j=1,2$. The boundary conditions in problem (3.3) provides a linear system of 4 equations with 4 unknowns which admits a unique solution if and only if its determinant $d$ is different from zero. We have

$$
d=4 e^{2 \rho \omega} \sin (1-i \rho) \omega \sin (1+i \rho) \omega
$$

Hence $d \neq 0$ for all $\xi \in \mathbb{R}$ if and only if

$$
r \neq \frac{k \pi}{\omega}+1, \forall k \in \mathbb{Z} \text { and } r \neq \frac{k \pi}{\omega}-1, \forall k \in \mathbb{Z}
$$

Under this assumption, the solution of (3.3) is given by

$$
\begin{aligned}
& v_{1}(\xi, y)=\frac{1}{2 \rho} \int_{0}^{\omega}\left[e^{\rho|y-z|}+e^{\rho(y+z)}\right] g_{1}(\xi, z) d z \\
& +\frac{1}{2 \rho d} \int_{0}^{\omega}\left[e^{\rho(2 \omega+y-z)}-e^{\rho(2 \omega-y-z)}\right]\left[\left(\cos 2 \omega-e^{2 \rho \omega}\right) g_{1}(\xi, z)+\sin 2 \omega g_{2}(\xi, z)\right] d z \\
& -\frac{1}{2 \rho d} \int_{0}^{\omega}\left[e^{\rho(2 \omega+y+z)}-e^{\rho(2 \omega-y+z)}\right]\left[\left(\cos 2 \omega+e^{2 \rho \omega}\right) g_{1}(\xi, z)-\sin 2 \omega g_{2}(\xi, z)\right] d z
\end{aligned}
$$




$$
\begin{aligned}
& v_{2}(\xi, y)=\frac{1}{2 \rho} \int_{0}^{\omega}\left[e^{\rho|y-z|}+e^{\rho(y+z)}\right] g_{2}(\xi, z) d z \\
& -\frac{1}{2 \rho d} \int_{0}^{\omega}\left[e^{\rho(2 \omega+y-z)}-e^{\rho(2 \omega-y-z)}\right]\left[\sin 2 \omega g_{1}(\xi, z)-\left(\cos 2 \omega-e^{2 \rho \omega}\right) g_{2}(\xi, z)\right] d z \\
& +\frac{1}{2 \rho d} \int_{0}^{\omega}\left[e^{\rho(2 \omega+y+z)}-e^{\rho(2 \omega-y+z)}\right]\left[\sin 2 \omega g_{1}(\xi, z)-\left(\cos 2 \omega-e^{2 \rho \omega}\right) g_{2}(\xi, z)\right] d z .
\end{aligned}
$$

\subsection{2. $L^{p}$ bounds for the solution}

We shall now use the last two identities to show the existence of a constant $C$ such that

$$
\|\boldsymbol{u}\|_{0, p, B} \leq C\|\boldsymbol{f}\|_{0, p, B} .
$$

For this purpose, we need the following lemma (see [15, Lemma 4.2.1.3]).

Lemma 3.1. Let $\xi, y, z \mapsto K(\xi, y, z)$ be a smooth (in $\xi)$ function such that

$$
\max _{y \in] 0, \omega[} \int_{0}^{\omega} \max _{\xi \in \mathbb{R}}\left\{|K(\xi, y, z)|+|\xi|\left|\partial_{\xi} K(\xi, y, z)\right|\right\} d z<+\infty
$$

and

$$
\max _{z \in] 0, \omega[} \int_{0}^{\omega} \max _{\xi \in \mathbb{R}}\left\{|K(\xi, y, z)|+\left|\xi \| \partial_{\xi} K(\xi, y, z)\right|\right\} d y<+\infty ;
$$

then the mapping $f \mapsto u$ defined by

$$
\hat{u}(\xi, y)=\int_{0}^{\omega} K(\xi, y, z) \hat{f}(\xi, z) d z
$$

is continuous in $L^{p}(B)$ for $p$ such that $1<p<\infty$.

Now we have to check that each term of the right hand side of (3.5) and of (3.6) satisfies the conditions (3.8) and (3.9) from the previous lemma. Indeed, we can see that the kernels involved in the expression of $v_{i}$ have the form $\frac{e^{\rho \beta}}{\rho}$ or $\frac{e^{\rho \beta}}{\rho d}$, where $\beta$ is a function depending only on the variables $y$ and $z$ such that

$$
\beta(y, z)=|y-z| \text { or } \beta(y, z)=a y+b z+c \geq 0 \quad \forall y, z \in[0, \omega],
$$

where $a, b \in\{-1,1\}$ and $c \in\{0,2 \omega, 4 \omega\}$.

Explicit calculations lead to

$$
d \geq e^{-2 \xi \omega} \cosh ^{2} \xi \omega|\sin \omega(1+r) \sin \omega(1-r)| \geq c,
$$

where

$$
c=\frac{|\sin \omega(1+r) \sin \omega(1-r)|}{4} .
$$


Let us set

$$
K_{1}(\xi, y, z)=\frac{e^{\rho \beta}}{\rho}, \quad K_{2}(\xi, y, z)=\frac{e^{\rho \beta}}{\rho d} .
$$

It is easy to check that

$$
\left|K_{1}(\xi, y, z)\right| \leq \frac{1}{r}, \quad|\xi|\left|\partial_{\xi} K_{1}(\xi, y, z)\right| \leq \beta+\frac{1}{r}, \quad\left|K_{2}(\xi, y, z)\right| \leq \frac{1}{r c} .
$$

In addition, we have

$$
|\xi|\left|\partial_{\xi} K_{2}(\xi, y, z)\right| \leq \frac{1}{c}\left\{\left(\beta+\frac{1}{r}\right)+\left|\frac{d^{\prime}}{d}\right|\right\} .
$$

It remains to estimate $\frac{d^{\prime}}{d}$. We compute it explicitly and obtain

$$
\begin{aligned}
\left|\frac{d^{\prime}}{d}\right| & =\left|2 \omega-i \omega \frac{\sin (2 r \omega+2 i \xi \omega)}{\sin [(1+r) \omega+i \xi \omega] \sin [(1-r) \omega-i \xi \omega]}\right| \\
& \leq 2 \omega\left(1+\frac{1}{c}\right) .
\end{aligned}
$$

It follows that (3.8) and (3.9) hold for each kernel involved in (3.5) and (3.6) and consequently the estimate (3.7) holds.

\subsection{3. $W^{2, p}$ bounds of the solution}

Now we are able to state the a priori estimate (3.2).

Theorem 3.2. Under the hypothesis (3.4), there exists a constant $c_{p}>0$ such that (3.2) holds for all $\boldsymbol{u} \in W^{2, p}(B)^{2}$ solution of (3.1).

Proof. Let $\eta_{\alpha}(\alpha=0, \omega)$ be two cut-off functions such that

(a) $\eta_{\alpha} \in C^{\infty}(\mathbb{R})$ with a compact support,

(b) $\eta_{0}=1$ (respectively $\eta_{\omega}=1$ ) in a neighbourhood of $F_{0}$ (respectively $F_{\omega}$ ) and vanishes near $F_{\omega}$ (respectively $\left.F_{0}\right)$.

(c) $\eta_{0}+\eta_{\omega}=1$.

Let us set $\boldsymbol{w}_{\alpha}=\left(w_{\alpha 1}, w_{\alpha 2}\right)^{\top}=\eta_{\alpha} \boldsymbol{u}$. Then the function $\boldsymbol{w}_{\alpha}$ is solution of the next boundary value problem on $\mathbb{R}_{\alpha,+}^{2}$ (with $\mathbb{R}_{0,+}^{2}=\mathbb{R}_{+}^{2}=(0, \infty) \times \mathbb{R}$ and $\mathbb{R}_{\omega,+}^{2}=$ $(-\infty, \omega) \times \mathbb{R})$ :

$$
\begin{aligned}
L w_{\alpha i} & =g_{\alpha i} & & \text { in } \mathbb{R}_{\alpha,+}^{2}, \\
w_{\alpha 1}(x, \alpha) \cos \alpha+w_{\alpha 2}(x, \alpha) \sin \alpha & =0 & & \forall x \in \mathbb{R}, \\
\sin \alpha \partial_{y} w_{\alpha 1}(x, \alpha)-\cos \alpha \partial_{y} w_{\alpha 2}(x, \alpha) & =0 & & \forall x \in \mathbb{R},
\end{aligned}
$$

where

$$
g_{\alpha i}=\eta_{\alpha} L u_{i}+2 \partial_{y} \eta_{\alpha} \partial_{y} u_{i}+\partial_{y}^{2} \eta_{\alpha} u_{i}, \quad i=1,2
$$


This problem can be looked as a system of partial differential equations as follows (see [19])

$$
\sum_{i=1}^{2} L_{i j}(x, \partial) w_{\alpha j}=g_{\alpha i} \text { in } \mathbb{R}_{\alpha,+}^{2}, \forall i=1,2,
$$

where $L_{i j}=\delta_{i j} L$.

It is clear that the system (3.13) is properly elliptic in $\mathbb{R}^{2}$ in the sense of Douglis-Nirenberg. Moreover we easily check that the boundary conditions (3.11), (3.12) cover the system (3.10) on $\mathbb{R}$. Therefore applying Theorem 10.5 of [1] (see also [19, Corollary 1.48]), we conclude the existence of a constant $C_{\alpha}>0$ such that

$$
\sum_{i=1}^{2}\left\|w_{\alpha i}\right\|_{2, p, B} \leq C_{\alpha}\left\{\sum_{i=1}^{2}\left\|g_{\alpha i}\right\|_{0, p, B}+\sum_{i=1}^{2}\left\|w_{\alpha i}\right\|_{0, p, B}\right\} .
$$

Adding this estimate for $\alpha=0$ with the one for $\alpha=\omega$ and using an interpolation inequality, there exists a constant $C>0$ such that

$$
\sum_{i=1}^{2}\left\|u_{i}\right\|_{2, p, B} \leq C \sum_{i=1}^{2}\left\{\left\|f_{i}\right\|_{0, p, B}+\left\|u_{i}\right\|_{0, p, B}\right\},
$$

which leads with (3.7) to the estimate (3.2).

\subsection{Bounds in a polygon}

Let us denote by $P^{2, p}(\Omega)$ the weighted Sobolev space of all distributions $u \in$ $\mathcal{D}^{\prime}(\Omega)$ satisfying

$$
r^{|\alpha|-2} D^{\alpha} u \in L^{p}(\Omega)
$$

for all $|\alpha| \leq 2$, where $r(x, y)$ denotes the distance from the point $(x, y)$ to the vertices of $\Omega . P^{2, p}(\Omega)$ is a Banach space for the norm

$$
\|u\|_{P^{2, p}(\Omega)}=\left(\sum_{\mid \alpha \leq 2}\left\|r^{|\alpha|-2} D^{\alpha} u\right\|_{0, p, \Omega}^{p}\right)^{\frac{1}{p}} .
$$

Theorem 3.3. Assume that

$$
3-\frac{2}{p} \neq \frac{k \pi}{\omega_{j}}, \forall k \in \mathbb{Z} \text { and } 1-\frac{2}{p} \neq \frac{k \pi}{\omega_{j}}, \forall k \in \mathbb{Z}, j=1, \ldots, N .
$$

Then there exists a constant $C_{p}>0$ such that

$$
\|\boldsymbol{E}\|_{P^{2, p}(\Omega)^{2}} \leq C_{p}\left\{\|\boldsymbol{J}\|_{0, p, \Omega}+\|\boldsymbol{E}\|_{1, p, \Omega}\right\},
$$

for every solution $\boldsymbol{E} \in P^{2, p}(\Omega)^{2}$ of (2.3)-(2.5). 
Proof. Let us fix a partition of unity $\left\{\eta_{j}\right\}, j=0, \ldots, N$ on $\bar{\Omega}$ such that

$$
\sum_{j=0}^{N} \eta_{j}=1
$$

and satisfying the following assumptions:

(a) the function $\eta_{j}$ belongs to $\mathcal{D}\left(\mathbb{R}^{2}\right)$ and fulfills the boundary conditions $\partial_{n} \eta_{j}=0$ on $\Gamma$.

(b) the support of $\eta_{0}$ does not contain any of the vertices of $\Omega$.

(c) the support of $\eta_{j}(j \neq 0)$ contains $S_{j}$ and does not contain any other vertex; in addition it does not intersect $\bar{\Gamma}_{k}$ for $k \neq j$ and $k \neq j+1$.

By [1, Theorem 10.5] (see also [19, Theorem 1.49]), there exists a positive constant $C_{0}$ such that

$$
\left\|\eta_{0} E_{i}\right\|_{2, p, \Omega} \leq C_{0}\left\{\left\|\Delta\left(\eta_{0} E_{i}\right)\right\|_{0, p, \Omega}+\left\|\eta_{0} E_{i}\right\|_{0, p, \Omega}\right\} .
$$

It remains to estimate $\eta_{j} \boldsymbol{E}$ for $j=1, \ldots, N$. For that purpose we fix $j$ and use local polar coordinates with origin at $S_{j}$, and such that $\theta=0$ on $\Gamma_{j+1}$ while $\theta=\omega_{j}$ on $\Gamma_{j}$. We denote by $G$ the infinite sector defined by

$$
G=\left\{(r \cos \theta, r \sin \theta) ; r>0,0<\theta<\omega_{j}\right\} .
$$

With this notation, the function $\boldsymbol{F}=\widetilde{\eta_{j} \boldsymbol{E}}$ (the extension by zero of $\eta_{j} \boldsymbol{E}$ outside the support of $\eta_{j}$ ) satisfies (2.4) and (2.5), as well as

$$
\left\|\Delta F_{i}\right\|_{0, p, G} \leq K\left\{\left\|J_{i}\right\|_{0, p, \Omega}+\left\|E_{i}\right\|_{1, p, \Omega}\right\}, i=1,2 .
$$

In polar coordinates, the boundary condition (2.4) takes the form

$$
E_{1}=0 \text { for } \theta=0, \quad E_{1} \cos \omega_{j}+E_{2} \sin \omega_{j}=0 \text { for } \theta=\omega_{j}
$$

while (2.5) becomes

$$
\partial_{\theta} E_{2}=0, \text { for } \theta=0, \quad \sin \omega_{j} \partial_{\theta} E_{1}-\cos \omega_{j} \partial_{\theta} E_{2}=0, \text { for } \theta=\omega_{j} .
$$

Let us set for $i=1,2$

$$
\begin{aligned}
& W_{i}(t, \theta)=e^{-\frac{2 t}{q}} F_{i}\left(e^{t}, \theta\right), \\
& K_{i}(t, \theta)=e^{\frac{2 t}{p}}\left(\Delta F_{i}\right)\left(e^{t}, \theta\right) .
\end{aligned}
$$

Then $\boldsymbol{W}=\left(W_{1}, W_{2}\right)^{\top}$ is solution of the following boundary value problem in the strip $B=\mathbb{R} \times] 0, \omega_{j}[$

$$
\begin{cases}\partial_{t}^{2} W_{i}+D_{\theta}^{2} W_{i}+\frac{4}{q} \partial_{t} W_{i}+\frac{4}{q^{2}} W_{i}=K_{i} & \text { in } B, i=1,2, \\ W_{1}=\partial_{\theta} W_{2}=0 & \text { on } \theta=0, \\ W_{1} \cos \omega_{j}+W_{2} \sin \omega_{j}=0 & \text { on } \theta=\omega_{j}, \\ \sin \omega_{j} \partial_{\theta} W_{1}-\cos \omega_{j} \partial_{\theta} W_{2}=0 & \text { on } \theta=\omega_{j} .\end{cases}
$$


In other words, $W$ is solution of problem (3.1) (studied in the previous section) with $r=\frac{2}{q}$ and $\omega=\omega_{j}$. Therefore, by Theorem 3.2, there exits a constant $C_{p}>0$ such that

$$
\sum_{i=1}^{2}\left\|W_{i}\right\|_{2, p, B} \leq C_{p} \sum_{i=1}^{2}\left\|K_{i}\right\|_{0, p, B} .
$$

Performing the inverse change of variables in (3.18) and (3.19) leads to

$$
\sum_{i=1}^{2}\left\|F_{i}\right\|_{P^{2, p}(\Omega)} \leq C_{p} \sum_{i=1}^{2}\left\|\Delta F_{i}\right\|_{0, p, \Omega},
$$

This last inequality together with (3.16) and (3.17) imply (3.15).

Now, we shall derive the consequences of the estimate (3.15). Let us set

$$
F_{p}=\left\{\boldsymbol{E} \in P^{2, p}(\Omega)^{2} \text { satisfying (2.4) and (2.5) }\right\},
$$

which is a Banach space for the norm of $P^{2, p}(\Omega)^{2}$. We also introduce the operator

$$
B_{p}: F_{p} \longrightarrow L^{p}(\Omega)^{2}: \boldsymbol{E} \longmapsto \Delta \boldsymbol{E} .
$$

Proposition 3.4. Assume that (3.14) holds, then the operator $B_{p}$ has a finite-dimensional kernel and a closed range.

Proof. Consequence of Theorem 3.3 and of Peetre's Lemma.

\section{The Fredholm alternative}

\subsection{The kernel}

Proposition 4.1. $\operatorname{ker} B_{p}=\{0\}$.

Proof. $\boldsymbol{E} \in \operatorname{ker} B_{p}$ means that $\boldsymbol{E} \in F_{p}$ and $\Delta \boldsymbol{E}=0$. Applying Green's formula, we get

$$
\int_{\Omega}(\operatorname{curl} \boldsymbol{E})^{2} d x+\int_{\Omega}(\operatorname{div} \boldsymbol{E})^{2} d x=0,
$$

which implies that

$$
\operatorname{curl} \boldsymbol{E}=0, \operatorname{div} \boldsymbol{E}=0 \text { in } \Omega .
$$

Therefore there exists $\phi \in H_{0}^{1}(\Omega)$ such that $\boldsymbol{E}=\operatorname{grad} \phi$. Since $\operatorname{div} \boldsymbol{E}=0$ in $\Omega$, we conclude that $\phi$ is harmonic. Hence $\phi=0$ and then $\boldsymbol{E}=\operatorname{grad} \phi=0$. 


\subsection{The range}

In this subsection, we shall study the range of $B_{p}$. Since the operator is closed, we shall instead investigate its annihilator $N_{q}$ which is a subspace of $L^{q}(\Omega)^{2}\left(\frac{1}{p}+\frac{1}{q}=1\right)$.

Let us recall that

$$
\begin{gathered}
N_{q}=\left\{\boldsymbol{F} \in L^{q}(\Omega)^{2} \text { satisfying (4.1) below }\right\} . \\
\int_{\Omega} \Delta \boldsymbol{E} \cdot \boldsymbol{F} d x d y=0 \quad \forall \boldsymbol{E} \in F_{p} .
\end{gathered}
$$

Lemma 4.2. Let $\boldsymbol{F} \in N_{q}$, then $\boldsymbol{F}$ is solution of

$$
\begin{array}{ll}
\Delta \boldsymbol{F}=\boldsymbol{0} & \text { in } \Omega, \\
\boldsymbol{F} \times \boldsymbol{n}=0 & \text { on } \Gamma, \\
\operatorname{div} \boldsymbol{F}=0 & \text { on } \Gamma .
\end{array}
$$

Proof. (4.2) follows by applying (4.1) with $\boldsymbol{E} \in \mathcal{D}(\Omega)^{2}$, we get

$$
\int_{\Omega} \Delta \boldsymbol{E} \cdot \boldsymbol{F} d x d y=0, \forall \boldsymbol{E} \in \mathcal{D}(\Omega)^{2},
$$

and consequently $\Delta F_{i}=0, i=1,2$, in the distributional sense.

Now for a fixed $1 \leq j \leq N$, given $\varphi_{j} \in \tilde{W}^{2-\frac{1}{p}, p}\left(\Gamma_{j}\right)$ and $\psi_{j} \in \tilde{W}^{1-\frac{1}{p}, p}\left(\Gamma_{j}\right)$, Lemma 5.2 below ensures the existence of $\boldsymbol{E} \in P^{2, p}(\Omega)^{2}$ such that (see Section 5 for the definitions and properties used in this proof)

$$
\begin{aligned}
& \boldsymbol{E} \cdot \boldsymbol{n}=\varphi_{j}, \quad \boldsymbol{E} \times \boldsymbol{n}=0, \quad \operatorname{curl} \boldsymbol{E}=\psi_{j}, \quad \operatorname{div} \boldsymbol{E}=0 \quad \text { on } \Gamma_{j}, \\
& \boldsymbol{E}=\boldsymbol{0}, \quad \operatorname{curl} \boldsymbol{E}=0, \quad \operatorname{div} \boldsymbol{E}=0 \quad \text { on } \Gamma_{k}, \forall k \neq j .
\end{aligned}
$$

Consequently, $\boldsymbol{E}$ belongs to $F_{p}$ and then $\int_{\Omega} \Delta \boldsymbol{E} \cdot \boldsymbol{F} d x d y=0$, for $F \in N_{q}$. Applying Corollary 5.4 to this function $\boldsymbol{E}$ and to $\boldsymbol{F} \in N_{q}$, we obtain

$$
\langle\operatorname{curl} \boldsymbol{E}, \boldsymbol{F} \times \boldsymbol{n}\rangle_{\Gamma_{j}}+\langle\boldsymbol{E} \cdot \boldsymbol{n}, \operatorname{div} \boldsymbol{F}\rangle_{\Gamma_{j}}=0,
$$

i.e.,

$$
\left\langle\psi_{j}, \boldsymbol{F} \times \boldsymbol{n}\right\rangle_{\Gamma_{j}}+\left\langle\varphi_{j}, \operatorname{div} \boldsymbol{F}\right\rangle_{\Gamma_{j}}=0 .
$$

If we let $\psi_{j}$ vary in $\tilde{W}^{1-\frac{1}{p}, p}\left(\Gamma_{j}\right)$ and $\varphi_{j}$ vary in $\tilde{W}^{2-\frac{1}{p}, p}\left(\Gamma_{j}\right)$, we obtain $(4.3)$ and (4.4).

Let us set

$$
M_{q}=\left\{\boldsymbol{E} \in L^{q}(\Omega)^{2} \text { satisfying (4.2) - (4.4) }\right\} .
$$

Lemma 4.2 shows that $N_{q}$ is a subspace of $M_{q}$, the next lemma ensures that these two spaces coincide. In other words, $N_{q}$ is completely characterized as the subspace of $L^{q}(\Omega)^{2}$ of the solutions of the adjoint problem (4.2)-(4.4). 
Lemma 4.3. $N_{q}=M_{q}$.

Proof. Let $\boldsymbol{F} \in M_{q}$ and $\boldsymbol{E} \in F_{p}$. Therefore $\boldsymbol{F} \in D\left(\Delta, L^{p}(\Omega)\right)^{2}$ and thus we can apply Corollary 5.4 to get

$$
\int_{\Omega} \Delta \boldsymbol{E} \cdot \boldsymbol{F} d x d y=0
$$

We shall now study the behavior of the elements of $M_{q}$. First of all, we show that they are regular far from the vertices.

Lemma 4.4. Let $\boldsymbol{F} \in M_{q}$, then $\boldsymbol{F} \in C^{\infty}(\bar{\Omega} \backslash V)^{2}$, where $V$ is any neighborhood of the vertices of $\Omega$.

Proof. The smoothness of $\boldsymbol{F}$ inside $\Omega$ follows from the fact that $F_{i}$ is a harmonic function in $\Omega$ for each $i=1,2$. It remains to prove that $\boldsymbol{F}$ is also smooth near any of the $\Gamma_{j}$. For that purpose, we proceed as in [15, proof of Lemma 4.4.2.1]: We fix $j$ and perform a change of coordinates such that $\Gamma_{j}$ is on the axis $\{y=0\}$ and $\Omega$ is above $\Gamma_{j}$. Then we introduce a cut-off function $\varphi \in C^{\infty}(\bar{\Omega})$, whose support does not intersect any of the sides $\bar{\Gamma}_{k}$ with $k \neq j$. In addition, $\varphi$ does not depend on $y$ for small values of $y$. Let us set $\boldsymbol{W}=\widetilde{\varphi \boldsymbol{F}}$. The function $\boldsymbol{W}$ belongs to $L^{q}\left(\mathbb{R}_{+}^{2}\right)$ and is solution of

$$
\begin{cases}-\Delta W_{i}+W_{i}=f_{i} & \text { in } \mathbb{R}_{+}^{2}, \\ W_{1}=0 & \text { on }\{y=0\}, \\ \frac{\partial W_{2}}{\partial y}=0 & \text { on }\{y=0\},\end{cases}
$$

where

$$
f_{i}=\left\{\varphi F_{i}-2 \nabla \varphi \nabla F_{i}-(\Delta \varphi) F_{i}\right\} .
$$

We remark that the boundary conditions in $W_{1}, W_{2}$ are decoupled. Therefore, each of the functions $W_{1}$ and $W_{2}$ can be looked as a solution of a boundary value problem which is similar to the one in Lemma 4.4.2.1 in [15]; so we deduce that $W_{i} \in$ $C^{\infty}(\bar{\Omega} \backslash V)$, for $i=1,2$.

In order to specify the behavior of $v \in M_{q}$ near the vertex $S_{j}$, we define the unbounded operator $\Lambda_{j}$ in $H_{j}=L^{2}(] 0, \omega_{j}[)^{2}$ as follows

$$
\Lambda_{j} \varphi=-\varphi^{\prime \prime},
$$

where $\varphi \in D\left(\Lambda_{j}\right)$, the domain of $\Lambda_{j}$ being given by

$$
\begin{gathered}
D\left(\Lambda_{j}\right)=\left\{\boldsymbol{\varphi}=\left(\varphi_{1}, \varphi_{2}\right)^{\top} \in H^{2}(] 0, \omega_{j}[)^{2} \text { satisfying (4.5) below }\right\} \\
\qquad \begin{array}{l}
\varphi_{1}(0)=\varphi_{2}^{\prime}(0)=0, \\
\cos \omega_{j} \varphi_{1}\left(\omega_{j}\right)+\sin \omega_{j} \varphi_{2}\left(\omega_{j}\right)=0 \\
\sin \omega_{j} \varphi_{1}^{\prime}\left(\omega_{j}\right)-\cos \omega_{j} \varphi_{2}^{\prime}\left(\omega_{j}\right)=0 .
\end{array}
\end{gathered}
$$


This is a nonnegative self-adjoint operator with a discrete spectrum. Let us denote by $\varphi_{j}$ an eigenfunction and by $\lambda_{j}^{2}$ the corresponding eigenvalue. We thus have $\varphi_{j} \in D\left(\Lambda_{j}\right)$ with

$$
-\varphi_{j, i}^{\prime \prime}=\lambda_{j}^{2} \varphi_{j, i}, \quad i=1,2 .
$$

By solving the last differential equation and taking into account the boundary conditions (4.5), we get

$$
\boldsymbol{\varphi}_{j, m}(\theta)=\left(\sin \left(\lambda_{j, m} \theta\right),-\cos \left(\lambda_{j, m} \theta\right)\right)^{\top}, \quad \lambda_{j, m}-1=\frac{m \pi}{\omega_{j}}, \quad m \in \mathbb{Z},
$$

or

$$
\tilde{\boldsymbol{\varphi}}_{j, m}(\theta)=\left(\sin \left(\tilde{\lambda}_{j, m} \theta\right), \cos \left(\tilde{\lambda}_{j, m} \theta\right)\right)^{\top}, \quad \tilde{\lambda}_{j, m}+1=\frac{m \pi}{\omega_{j}}, \quad m \in \mathbb{Z} .
$$

Remark 4.5. For covering all the linearly independent eigenfunctions of $\Lambda_{j}$, it suffices to consider only the functions $\boldsymbol{\varphi}_{j, m}$ corresponding to $\lambda_{j, m}$. Indeed we may notice that $\tilde{\lambda}_{j, m}=-\lambda_{j,-m}$ and that $\tilde{\boldsymbol{\varphi}}_{j, m}=-\varphi_{j,-m}$. This means that

$$
\operatorname{Sp}\left(\Lambda_{j}\right)=\left\{\lambda^{2}: \lambda-1 \in E_{j}\right\}
$$

where

$$
E_{j}=\left\{\frac{m \pi}{\omega_{j}}, \quad m \in \mathbb{Z}\right\} .
$$

The following theorem gives near each corner $S_{j}$, an expansion for the elements of $M_{q}$ in series of the eigenfunctions of $\Lambda_{j}$.

Theorem 4.6. If $v \in M_{q}$, then for all $j \in\{1, \ldots, N\}$, and for every $\rho>0$ fixed sufficiently small,

$$
\begin{aligned}
\boldsymbol{v}\left(r_{j} e^{i \theta_{j}}\right)= & \sum_{\lambda_{j, m}>\frac{-2}{q}} \alpha_{j, m} r_{j}^{\lambda_{j, m}} \boldsymbol{\varphi}_{j, m}\left(\theta_{j}\right) \\
& +\sum_{\lambda_{j, m}<\frac{2}{q}} \tilde{\alpha}_{j, m} r_{j}^{-\lambda_{j, m}} \boldsymbol{\varphi}_{j, m}\left(\theta_{j}\right) \text { in } D_{j, \rho},
\end{aligned}
$$

where $\left(r_{j}, \theta_{j}\right)$ denotes the polar coordinates with origin at $S_{j}, D_{j, \rho}=\left\{r_{j} e^{i \theta_{j}}\right.$ : $\left.0<r_{j}<\rho, 0<\theta_{j}<\omega_{j}\right\}, \alpha_{j, m}$ and $\tilde{\alpha}_{j, m}$ are real numbers given by $\alpha_{j, m} r_{j}^{\lambda_{j, m}}+\tilde{\alpha}_{j, m} r_{j}^{-\lambda_{j, m}}=\int_{0}^{\omega_{j}}\left\{v_{1}\left(r_{j} e^{i \theta_{j}}\right) \sin \left(\lambda_{j, m} \theta_{j}\right)-v_{2}\left(r_{j} e^{i \theta_{j}}\right) \cos \left(\lambda_{j, m} \theta_{j}\right)\right\} d \theta_{j}$.

In addition, there exists a constant $L>0$ depending only on $v$ and $\rho$ such that

$$
\left|\alpha_{j, m}\right| \leq L \rho^{-\lambda_{j, m}-\frac{2}{q}}\left(\lambda_{j, m} q+2\right)^{\frac{1}{q}}
$$


when $\lambda_{j, m} \geq \frac{2}{q}$ and

$$
\left|\tilde{\alpha}_{j, m}\right| \leq L \rho^{\lambda_{j, m}-\frac{2}{q}}\left(-\lambda_{j, m} q+2\right)^{\frac{1}{q}}
$$

when $\lambda_{j, m} \leq \frac{-2}{q}$.

Proof. Let us fix $j \in\{1,2, \ldots, N\}$ and for shortness drop the index $j$. Thanks to Lemma 4.4, $v$ is smooth for $r>0$ and consequently the function

$$
\theta \longmapsto \boldsymbol{v}\left(r e^{i \theta}\right)
$$

is differentiable in $r$ with values in $H_{j}=L^{2}(] 0, \omega_{j}[)^{2}$, for each $\left.\left.r \in\right] 0, \rho\right], \rho>0$ chosen sufficiently small.

Writing (4.2)-(4.4) in polar coordinates, we notice that for each $r \in] 0, \rho]$, $v\left(r e^{i \theta}\right) \in D\left(\Lambda_{j}\right)$ and that $v$ is solution of

$$
\frac{\partial^{2} \boldsymbol{v}}{\partial r^{2}}+\frac{1}{r} \frac{\partial \boldsymbol{v}}{\partial r}-\frac{1}{r^{2}} \Lambda_{j} v=0, \quad 0<r<\rho .
$$

Since the sequence $\left(\boldsymbol{\varphi}_{j, m}\right)$ is a basis of $H_{j}, \boldsymbol{v}$ can be expanded in the series

$$
\boldsymbol{v}\left(r e^{i \theta}\right)=\sum_{\substack{m \in \mathbb{Z} \\ \lambda_{j, m}-1=\frac{m \pi}{\omega_{j}}}} v_{m}(r) \boldsymbol{\varphi}_{j, m}(\theta),
$$

where

$$
v_{m}(r)=\int_{0}^{\omega_{j}}\left\{v_{1}\left(r e^{i \theta}\right) \sin \lambda_{j, m} \theta-v_{2}\left(r e^{i \theta}\right) \cos \lambda_{j, m} \theta\right\} d \theta .
$$

However, equation (4.9) implies that

$$
v_{m}^{\prime \prime}(r)+\frac{1}{r} v_{m}^{\prime}(r)-\frac{\lambda_{j, m}^{2}}{r^{2}} v_{m}(r)=0, \quad 0<r<\rho .
$$

Consequently, we have

$$
v_{m}(r)=\alpha_{j, m} r^{\lambda_{j, m}}+\tilde{\alpha}_{j, m} r^{-\lambda_{j, m}}
$$

because $\lambda_{j, m} \neq 0$.

As $v$ belongs to $L^{q}\left(D_{\rho}\right)^{2}$, it follows from identity (4.10) that

$$
\left|v_{m}(r)\right|^{q} \leq C\left\{\int_{0}^{\omega_{j}}\left|v_{1}\left(r e^{i \theta}\right)\right|^{q} d \theta+\int_{0}^{\omega_{j}}\left|v_{2}\left(r e^{i \theta}\right)\right|^{q} d \theta\right\},
$$


for some $C>0$ depending on $\omega_{j}$ and $p$, and consequently

$$
\int_{0}^{\rho}\left|v_{m}(r)\right|^{q} r d r \leq C\left\{\left\|v_{1}\right\|_{0, q, D_{\rho}}^{q}+\left\|v_{2}\right\|_{0, q, D_{\rho}}^{q}\right\} .
$$

This last inequality implies that $\alpha_{j, m}=0$ when $\lambda_{j, m} \leq \frac{-2}{q}$ and $\tilde{\alpha}_{j, m}=0$ when $\lambda_{j, m} \geq 2 / q$. In addition, we have

$$
\left|\alpha_{j, m}\right|^{q} \int_{0}^{\rho} r^{\lambda_{j, m} q+1} d r=\left|\alpha_{j, m}\right|^{q} \frac{\rho^{\lambda_{j, m} q+2}}{\lambda_{j, m} q+2} \leq C\left\{\left\|v_{1}\right\|_{0, q, D_{\rho}}^{q}+\left\|v_{2}\right\|_{0, q, D_{\rho}}^{q}\right\},
$$

for $\lambda_{j, m} \geq 2 / q$. This leads to (4.7). The same argument leads to (4.8).

Using Remark 4.5, (4.6) takes the equivalent form

Corollary 4.7. If $v \in M_{q}$, then for all $j \in\{1, \ldots, N\}$, and every $\rho>0$ fixed sufficiently small,

$$
\boldsymbol{v}\left(r e^{i \theta}\right)=\sum_{\lambda_{j, m}>\frac{-2}{q}} \alpha_{j, m} r^{\lambda_{j, m}} \boldsymbol{\varphi}_{j, m}(\theta)+\sum_{\tilde{\lambda}_{j, m}>\frac{-2}{q}} \tilde{\alpha}_{j, m} r^{\tilde{\lambda}_{j, m}} \tilde{\boldsymbol{\varphi}}_{j, m}(\theta) \text { in } D_{j, \rho},
$$

where $\alpha_{j, m}, \tilde{\alpha}_{j, m}$ are real numbers such that

$$
\left|\alpha_{j, m}\right| \leq L \rho^{-\lambda_{j, m}-\frac{2}{q}}\left(\lambda_{j, m} q+2\right)^{\frac{1}{q}}
$$

when $\lambda_{j, m} \geq \frac{2}{q}$ and

$$
\left|\tilde{\alpha}_{j, m}\right| \leq L \rho^{-\tilde{\lambda}_{j, m}-\frac{2}{q}}\left(\tilde{\lambda}_{j, m} q+2\right)^{\frac{1}{q}}
$$

when $\tilde{\lambda}_{j, m} \geq \frac{2}{q}$, for some $L>0$ depending only on $v$ and $\rho$.

Here again, we denote by $\eta_{j}$ a cut-off function which is 1 in a neighbourhood of $S_{j}$, whose support does not intersect $\bar{\Gamma}_{k}$ for $k \neq j$ and $j+1$ and such that $\partial_{n} \eta_{j}=0$ on $\Gamma$.

We shall try to give a basis of $M_{q}$ and thus deduce its dimension. For that purpose, we will correct the functions $r_{j}^{\lambda_{j, m}} \boldsymbol{\varphi}_{j, m}$ (respectively $r_{j}^{\tilde{\lambda}_{j, m}} \tilde{\boldsymbol{\varphi}}_{j, m}$ ) in the expansion (4.12) to get a generating family of $M_{q}$.

Lemma 4.8. i) For each $j$ and each $\lambda_{j, m}$ such that $\lambda_{j, m}-1=\frac{m \pi}{\omega_{j}} \in E_{j}$, with $\left.\left.\lambda_{j, m} \in\right]-\frac{2}{q}, 1\right)$, there exists a non zero element $\sigma_{j, m} \in M_{q}$ satisfying

$$
\sigma_{j, m}-\eta_{j} \boldsymbol{u}_{j, m} \in X_{N}(\Omega),
$$


where

$$
\boldsymbol{u}_{j, m}\left(r_{j} e^{i \theta_{j}}\right)=r^{\lambda_{j, m}} \boldsymbol{\varphi}_{j, m}(\theta)
$$

ii) If $p>2$, for each $j$ and each $\tilde{\lambda}_{j, m}$ such that $\tilde{\lambda}_{j, m}+1=\frac{m \pi}{\omega_{j}} \in E_{j}$, with $\left.\left.\lambda_{j, m} \in\right]-\frac{2}{q},-1\right]$, there exists a non zero element $\tilde{\sigma}_{j, m} \in M_{q}$ satisfying

$$
\tilde{\sigma}_{j, m}-\eta_{j} \tilde{\boldsymbol{u}}_{j, m} \in X_{N}(\Omega),
$$

where

$$
\tilde{\boldsymbol{u}}_{j, m}\left(r_{j} e^{i \theta_{j}}\right)=r^{\tilde{\lambda}_{j, m}} \tilde{\boldsymbol{\varphi}}_{j, m}\left(\theta_{j}\right) .
$$

Proof. i) Since $\boldsymbol{u}_{j, m}$ is harmonic and the cut-off function $\eta_{j}$ is constant near the origin, it is easy to check that

$$
\Delta\left(\eta_{j} \boldsymbol{u}_{j, m}\right)=\boldsymbol{f}_{j, m} \in C^{\infty}(\bar{\Omega})^{2},
$$

and that $\eta_{j} \boldsymbol{u}_{j, m}$ fulfills the boundary conditions (2.4) and (2.5).

Therefore by Proposition 2.1 there exists a unique solution $v_{j, m} \in X_{N}(\Omega)$ of

$$
\begin{cases}\Delta \boldsymbol{v}_{j, m}=\boldsymbol{f}_{j, m} & \text { in } \Omega, \\ \boldsymbol{v}_{j, m} \times \boldsymbol{n}=0 & \text { on } \Gamma, \\ \operatorname{div} \boldsymbol{v}_{j, m}=0 & \text { on } \Gamma .\end{cases}
$$

The claim follows by setting

$$
\sigma_{j, m}=\eta_{j} \boldsymbol{u}_{j, m}-\boldsymbol{v}_{j, m} .
$$

Indeed we can say that $\sigma_{j, m}$ is different from zero, since we easily check that $\eta_{j} \boldsymbol{u}_{j, m}$ belongs to $X_{N}(\Omega)$ if and only if $\lambda_{j, m}>0$, while

$$
\operatorname{div} \boldsymbol{u}_{j, m}=r^{\lambda_{j, m}-1} \psi_{j}\left(\theta_{j}\right),
$$

for some non zero smooth function $\psi_{j}$. Therefore $\operatorname{div}\left(\eta \boldsymbol{u}_{j, m}\right)$ belongs to $H^{1}(\Omega)$ if and only if $\lambda_{j, m} \geq 1$ (because for $\lambda_{j, m}=1, \boldsymbol{u}_{j, m}$ is a polynomial of order 1). Hence for $\lambda_{j, m}<1, \eta_{j} \boldsymbol{u}_{j, m}$ cannot be equal to $\boldsymbol{v}_{j, m}$, due to Lemma 2.2.

ii) The existence of a unique solution $\tilde{\boldsymbol{v}}_{j, m} \in X_{N}(\Omega)$ of

$$
\begin{cases}\Delta \tilde{\boldsymbol{v}}_{j, m}=\Delta\left(\eta_{j} \tilde{\boldsymbol{u}}_{j, m}\right) & \text { in } \Omega, \\ \tilde{\boldsymbol{v}}_{j, m} \times \boldsymbol{n}=0 & \text { on } \Gamma, \\ \operatorname{div} \tilde{\boldsymbol{v}}_{j, m}=0 & \text { on } \Gamma,\end{cases}
$$

follows as in the first item. Again the claim follows by setting

$$
\tilde{\sigma}_{j, m}=\eta_{j} \tilde{\boldsymbol{u}}_{j, m}-\tilde{\boldsymbol{v}}_{j, m} .
$$

Now as $\operatorname{div} \tilde{\boldsymbol{u}}_{j, m}=0$ and $\operatorname{curl} \tilde{\boldsymbol{u}}_{j, m}=0, \eta_{j} \tilde{\boldsymbol{u}}_{j, m}$ belongs to $X_{N}(\Omega)$ if and only if $\tilde{\lambda}_{j, m}>-1$, and $\operatorname{div}\left(\eta \tilde{\boldsymbol{u}}_{j, m}\right)$ always belongs to $H^{1}(\Omega)$. Consequently for $\tilde{\lambda}_{j, m} \leq$ $-1, \eta_{j} \tilde{\boldsymbol{u}}_{j, m}$ cannot be equal to $\tilde{\boldsymbol{v}}_{j, m}$. 
Theorem 4.9. The dimension of $M_{q}$ is equal to

$$
\begin{aligned}
\operatorname{dim} M_{q}= & \sum_{1 \leq j \leq N} \operatorname{card}\left\{m \in \mathbb{Z} ;-\frac{2}{q}<\lambda_{j, m}<1 \text { and } \lambda_{j, m}-1=\frac{m \pi}{\omega_{j}} \in E_{j}\right\} \\
& +\sum_{1 \leq j \leq N} \operatorname{card}\left\{m \in \mathbb{Z} ;-\frac{2}{q}<\tilde{\lambda}_{j, m} \leq-1 \text { and } \tilde{\lambda}_{j, m}+1=\frac{m \pi}{\omega_{j}} \in E_{j}\right\},
\end{aligned}
$$

with the convention that the second set is empty if $p \leq 2$.

Proof. Let $\boldsymbol{v} \in M_{q}$ and consider a fixed corner $S_{j}$. Corollary 4.7 shows that $\boldsymbol{v}$ admits the next expansion in a neighborhood of $S_{j}$ :

$$
\begin{aligned}
\boldsymbol{v}= & \sum_{\substack{\lambda_{j, m}-1 \in E_{j} \\
\lambda_{j, m} \geq 1}} \alpha_{j, m} r^{\lambda_{j, m}} \boldsymbol{\varphi}_{j, m}(\theta)+\sum_{\substack{\tilde{\lambda}_{j, m}+1 \in E_{j} \\
\tilde{\lambda}_{j, m}>-1}} \tilde{\alpha}_{j, m} r^{\tilde{\lambda}_{j, m}} \tilde{\boldsymbol{\varphi}}_{j, m}(\theta) \\
& +\sum_{\substack{\lambda_{j, m}-1 \in E_{j} \\
\frac{-2}{q}<\lambda_{j, m}<1}} \alpha_{j, m} \boldsymbol{u}_{j, m}+\sum_{\substack{\tilde{\lambda}_{j, m}+1 \in E_{j} \\
\frac{-2}{q}<\tilde{\lambda}_{j, m} \leq-1}} \tilde{\alpha}_{j, m} \tilde{\boldsymbol{u}}_{j, m} .
\end{aligned}
$$

Therefore, we deduce by Lemma 4.8 that

$$
\begin{gathered}
\boldsymbol{v}-\sum_{\substack{\lambda_{j, m}-1 \in E_{j} \\
\lambda_{j, m} \geq 1}} \alpha_{j, m} r^{\lambda_{j, m}} \boldsymbol{\varphi}_{j, m}(\theta)-\sum_{\substack{\tilde{\lambda}_{j, m}+1 \in E_{j} \\
\tilde{j}_{j, m}>-1}} \tilde{\alpha}_{j, m} r^{\tilde{\lambda}_{j, m}} \tilde{\boldsymbol{\varphi}}_{j, m}(\theta) \\
-\sum_{\substack{\lambda_{j, m}-1 \in E_{j} \\
\frac{-2}{q}<\lambda_{j, m}<1}} \alpha_{j, m} \sigma_{j, m}-\sum_{\substack{\tilde{\lambda}_{j, m}+1 \in E_{j} \\
\frac{-2}{q}<\tilde{\lambda}_{j, m} \leq-1}} \tilde{\alpha}_{j, m} \tilde{\sigma}_{j, m} \in X_{N}\left(D_{j, \rho}\right) .
\end{gathered}
$$

We shall now show that each series in (4.15) belongs to $X_{N}\left(D_{j, \rho_{1}}\right)$ where $\rho_{1}<\rho$. Indeed, let us denote the series in $\lambda_{j, m} \geq 1$ (respectively $\tilde{\lambda}_{j, m}>-1$ ) by $\boldsymbol{w}^{-}$ (respectively $\boldsymbol{w}^{+}$).

We clearly have

$$
\left|\operatorname{div} \boldsymbol{w}^{-}\right|+\left|\operatorname{curl} \boldsymbol{w}^{-}\right| \leq C \sum_{\lambda_{j, m} \geq 1}\left|\alpha_{j, m}\right| r_{j}^{\lambda_{j, m}-1}
$$

for some $C>0$. It follows from inequality (4.13) that

$$
\left|\operatorname{div} \boldsymbol{w}^{-}\right|+\left|\operatorname{curl} \boldsymbol{w}^{-}\right| \leq C L \sum_{\lambda_{j, m} \geq \frac{2}{q}} \frac{\left(\lambda_{j, m} q+2\right)^{\frac{1}{q}}}{\rho_{1} \rho^{\frac{2}{q}}}\left(\frac{\rho_{1}}{\rho}\right)^{\lambda_{j, m}}+C \sum_{1 \leq \lambda_{m}<\frac{2}{q}}\left|\alpha_{j, m}\right| r_{j}^{\lambda_{j, m}-1},
$$


and this last series is convergent since $\rho_{1}<\rho$. This allows to conclude that $\boldsymbol{w}^{-}$ belongs to $X_{N}\left(D_{j, \rho_{1}}\right)$. The same arguments apply to $\boldsymbol{w}^{+}$using the estimate (4.14). Consequently, we get

$$
\boldsymbol{v}-\sum_{\substack{\lambda_{j, m}-1 \in E_{j} \\ \frac{-2}{q}<\lambda_{j, m}<1}} \alpha_{j, m} \sigma_{j, m}-\sum_{\substack{\tilde{\lambda}_{j, m}+1 \in E_{j} \\ \frac{-2}{q}<\tilde{\lambda}_{j, m} \leq-1}} \tilde{\alpha}_{j, m} \tilde{\sigma}_{j, m} \in X_{N}\left(D_{\rho_{1}}\right) .
$$

This smoothness result is valid near each of the corners. By Lemma 4.4, we deduce that

$$
\boldsymbol{\varphi}:=\boldsymbol{v}-\sum_{j=1}^{N}\left\{\sum_{\substack{\lambda_{j, m}-1 \in E_{j} \\ \frac{-2}{q}<\lambda_{j, m}<1}} \alpha_{j, m} \sigma_{j, m}+\sum_{\substack{\tilde{\lambda}_{j, m}+1 \in E_{j} \\ \frac{-2}{q}<\tilde{\lambda}_{j, m} \leq-1}} \tilde{\alpha}_{j, m} \tilde{\sigma}_{j, m}\right\} \in X_{N}(\Omega) .
$$

As $\varphi$ belongs to $M_{q}$, it is a solution of problem (4.2)-(4.4) and since it also belongs to $X_{N}(\Omega)$, we conclude that $\varphi=0$. In other words, we have

$$
\boldsymbol{v}=\sum_{j=1}^{N}\left\{\sum_{\substack{\lambda_{j, m}-1 \in E_{j} \\ \frac{-2}{q}<\lambda_{j, m}<1}} \alpha_{j, m} \sigma_{j, m}+\sum_{\substack{\tilde{\lambda}_{j, m}+1 \in E_{j} \\ \frac{-2}{q}<\tilde{\lambda}_{j, m} \leq-1}} \tilde{\alpha}_{j, m} \tilde{\sigma}_{j, m}\right\}
$$

It remains to show that the functions $\sigma_{j, m}$ with $\frac{-2}{q}<\lambda_{j, m}<1$ and $\tilde{\sigma}_{j, m}$ with $\frac{-2}{q}<\tilde{\lambda}_{j, m} \leq-1$ are linearly independent. Let then assume that there exist $\alpha_{j, m}$ and $\tilde{\alpha}_{j, m}$ such that

$$
\sum_{j=1}^{N}\left\{\sum_{\substack{\lambda_{j, m}-1 \in E_{j} \\ \frac{-2}{q}<\lambda_{j, m}<1}} \alpha_{j, m} \sigma_{j, m}+\sum_{\substack{\tilde{\lambda}_{j, m}+1 \in E_{j} \\ \frac{-2}{q}<\tilde{\lambda}_{j, m} \leq-1}} \tilde{\alpha}_{j, m} \tilde{\sigma}_{j, m}\right\}=0
$$

Then by the construction from Lemma 4.8 we deduce that the function

$$
\boldsymbol{w}:=\sum_{j=1}^{N}\left\{\sum_{\substack{\lambda_{j, m}-1 \in E_{j} \\ \frac{-2}{q}<\lambda_{j, m}<1}} \alpha_{j, m} \eta_{j} \boldsymbol{u}_{j, m}+\sum_{\substack{\tilde{\lambda}_{j, m}+1 \in E_{j} \\ \frac{-2}{q}<\tilde{\lambda}_{j, m} \leq-1}} \tilde{\alpha}_{j, m} \eta_{j} \tilde{\boldsymbol{u}}_{j, m}\right\}
$$


belongs to $X_{N}(\Omega)$ with $\operatorname{div} \boldsymbol{w} \in H_{0}^{1}(\Omega)$. As the supports of the cut-off functions $\eta_{j}$ are supposed to be disjoint, for all $j=1, \cdots, N$, we obtain that

$$
\boldsymbol{w}_{j}:=\sum_{\substack{\lambda_{j, m}-1 \in E_{j} \\ \frac{-2}{q}<\lambda_{j, m}<1}} \alpha_{j, m} \eta_{j} \boldsymbol{u}_{j, m}+\sum_{\substack{\tilde{\lambda}_{j, m}+1 \in E_{j} \\ \frac{-2}{q}<\tilde{\lambda}_{j, m} \leq-1}} \tilde{\alpha}_{j, m} \eta_{j} \tilde{\boldsymbol{u}}_{j, m}
$$

belongs to $X_{N}\left(D_{j, \rho}\right)$ with $\operatorname{div} \boldsymbol{w}_{j} \in H_{0}^{1}\left(D_{j, \rho}\right)$. At this stage, as $\operatorname{div}\left(\eta_{j} \tilde{\boldsymbol{u}}_{j, m}\right)$ is regular, we obtain that

$$
\sum_{\substack{\lambda_{j, m}-1 \in E_{j} \\ \frac{-2}{q}<\lambda_{j, m}<1}} \alpha_{j, m} \operatorname{div} \boldsymbol{u}_{j, m} \in H^{1}\left(D_{j, \rho}\right) .
$$

As $\operatorname{div} \boldsymbol{u}_{j, m}$ behaves like $r^{\lambda_{j, m}-1}$ which does not belong to $H^{1}\left(D_{j, \rho}\right)$ for $\lambda_{j, m}<1$ and are linearly independent, we deduce that $\alpha_{j, m}=0$, for all $\frac{-2}{q}<\lambda_{j, m}<1$.

Hence $\boldsymbol{w}_{j}$ reduces to

$$
\boldsymbol{w}_{j}=\sum_{\substack{\tilde{\lambda}_{j, m}+1 \in E_{j} \\ \frac{-2}{q}<\tilde{\lambda}_{j, m} \leq-1}} \tilde{\alpha}_{j, m} \eta_{j} \tilde{\boldsymbol{u}}_{j, m} .
$$

Using the property $\boldsymbol{w}_{j} \in X_{N}\left(D_{j, \rho}\right)$ and the fact that $\tilde{\boldsymbol{u}}_{j, m}$ behaves like $r^{\tilde{\lambda}_{j, m}}$ which does not belong to $L^{2}\left(D_{j, \rho}\right)$ for $\tilde{\lambda}_{j, m} \leq-1$ and are linearly independent, we conclude that $\tilde{\alpha}_{j, m}=0$ for $\tilde{\lambda}_{j, m} \leq-1$.

The last result shows that $M_{q}$ is finite-dimensional, and as the kernel of $B_{p}$ is reduced to $\{0\}, B_{p}$ is a Fredholm operator from $F_{p}$ into $L^{p}(\Omega)^{2}$ of index equal to $-\operatorname{dim} M_{q}$. Hence we are looking for $\operatorname{dim} M_{q}$ functions which are not in the range of $B_{p}$. In accordance with [11, Section 3], for all $j=1, \cdots, N$ and all $\lambda_{j, m}-1 \in E_{j}$ such that $-\frac{2}{q}<\lambda_{j, m}<1$, we set

$$
\boldsymbol{S}_{j, m}\left(r_{j} e^{i \theta_{j}}\right)=\eta_{j}\left(r_{j}\right) r_{j}^{-\lambda_{j, m}} \boldsymbol{\varphi}_{j, m}\left(\theta_{j}\right) .
$$

Similarly for $p>2$, all $j=1, \cdots, N$ and all $\tilde{\lambda}_{j, m}+1 \in E_{j}$ such that $-\frac{2}{q}<$ $\tilde{\lambda}_{j, m} \leq-1$, we set

$$
\tilde{\boldsymbol{S}}_{j, m}\left(r_{j} e^{i \theta_{j}}\right)=\eta_{j}\left(r_{j}\right) r_{j}^{-\tilde{\lambda}_{j, m}} \tilde{\boldsymbol{\varphi}}_{j, m}\left(\theta_{j}\right) .
$$

Note that $\boldsymbol{S}_{j, m}$ is, up to a factor, the gradient of a singular function of the Laplace operator with Dirichlet boundary conditions (near $S_{j}$ ), while $\operatorname{div} \tilde{\boldsymbol{S}}_{j, m}$ is, up to a factor, such a singular function. 
We easily check the following

Lemma 4.10. i) For all $j=1, \cdots, N$ and all $\lambda_{j, m}-1 \in E_{j}$ such that $-\frac{2}{q}<$ $\lambda_{j, m}<1$, we have $\boldsymbol{S}_{j, m} \in X_{N}(\Omega) \backslash P^{2, p}(\Omega)^{2}$,

$$
\begin{cases}\Delta \boldsymbol{S}_{j, m} \in C^{\infty}(\bar{\Omega})^{2}, & \\ \boldsymbol{S}_{j, m} \times \boldsymbol{n}=0 & \text { on } \Gamma \\ \operatorname{div} \boldsymbol{S}_{j, m}=0 & \text { on } \Gamma\end{cases}
$$

and $\operatorname{div} \boldsymbol{S}_{j, m} \in W_{0}^{1, s}(\Omega)$, for all $s \geq 2$.

ii) For $p>2$, all $j=1, \cdots, N$ and all $\tilde{\lambda}_{j, m}+1 \in E_{j}$ such that $-\frac{2}{q}<\tilde{\lambda}_{j, m} \leq-1$, we have $\tilde{\boldsymbol{S}}_{j, m} \in X_{N}(\Omega) \backslash P^{2, p}(\Omega)^{2}$,

$$
\begin{cases}\Delta \tilde{\boldsymbol{S}}_{j, m} \in C^{\infty}(\bar{\Omega})^{2}, & \\ \tilde{\boldsymbol{S}}_{j, m} \times \boldsymbol{n}=0 & \text { on } \Gamma \\ \operatorname{div} \tilde{\boldsymbol{S}}_{j, m}=0 & \text { on } \Gamma\end{cases}
$$

and $\operatorname{div} \tilde{\boldsymbol{S}}_{j, m} \in H_{0}^{1}(\Omega)$.

Lemma 4.11. i) For all $j=1, \cdots, N$ and all $\lambda_{j, m}-1 \in E_{j}$ such that $-\frac{2}{q}<$ $\lambda_{j, m}<1, \Delta \boldsymbol{S}_{j, m}$ is not orthogonal to $N_{q}$.

ii) For $p>2$, all $j=1, \cdots, N$ and all $\tilde{\lambda}_{j, m}+1 \in E_{j}$ such that $-\frac{2}{q}<\tilde{\lambda}_{j, m} \leq-1$, $\Delta \tilde{\boldsymbol{S}}_{j, m}$ is not orthogonal to $N_{q}$.

Proof. i) We proceed by contradiction. If we assume that $\Delta \boldsymbol{S}_{j, m}$ is orthogonal to $N_{q}$, then there exists $\boldsymbol{w}_{j, m} \in P^{2, p}(\Omega)^{2}$ fulfilling (2.4) and (2.5) such that

$$
\Delta \boldsymbol{w}_{j, m}=\Delta \boldsymbol{S}_{j, m} .
$$

Therefore $\boldsymbol{w}_{j, m}-\boldsymbol{S}_{j, m}$ is a solution of the homogeneous problem (2.3)-(2.5) and belongs to $X_{N}(\Omega)$. The uniqueness of the variational solution implies that $\boldsymbol{S}_{j, m}$ belongs to $P^{2, p}(\Omega)^{2}$ which contradicts Lemma 4.10.

The second item is proved in the same way.

We are now ready to state the main result of our paper, namely the splitting of the solution of the variational problem (2.6) into a regular part and a singular one. 
Theorem 4.12. Let $p \geq 4 / 3$ and suppose that (3.14) holds. Then for each $\boldsymbol{J} \in$ $L^{p}(\Omega)^{2}$, there exist unique real numbers $c_{j, m}, \tilde{c}_{j, m}$ and a unique $\boldsymbol{E} \in X_{N}(\Omega)$ solution of the variational problem (2.6) such that

$$
\boldsymbol{E}-\sum_{j=1}^{N}\left\{\sum_{\substack{\lambda_{j, m}-1 \in E_{j} \\ \frac{-2}{q}<\lambda_{j, m}<1}} c_{j, m} \boldsymbol{S}_{j, m}-\sum_{\substack{\tilde{\lambda}_{j, m}+1 \in E_{j} \\ \frac{-2}{q}<\tilde{\lambda}_{j, m} \leq-1}} \tilde{c}_{j, m} \tilde{\boldsymbol{S}}_{j, m}\right\} \in P^{2, p}(\Omega)^{2} .
$$

Proof. The functions $\Delta \boldsymbol{S}_{j, m}$ and $\Delta \tilde{\boldsymbol{S}}_{j, m}$ are linearly independent and their number is exactly the codimension of the range of $B_{p}$. Hence $L^{p}(\Omega)^{2}$ is spanned by these functions and the range of $B_{p}$, i.e., any $\boldsymbol{J} \in L^{p}(\Omega)^{2}$ is uniquely written

$$
\boldsymbol{J}=\Delta \boldsymbol{E}_{R}+\sum_{j=1}^{N}\left\{\sum_{\substack{\lambda_{j, m}-1 \in E_{j} \\ \frac{-2}{q}<\lambda_{j, m}<1}} c_{j, m} \Delta \boldsymbol{S}_{j, m}-\sum_{\substack{\tilde{\lambda}_{j, m}+1 \in E_{j} \\ \frac{-2}{q}<\tilde{\lambda}_{j, m} \leq-1}} \tilde{c}_{j, m} \Delta \tilde{\boldsymbol{S}}_{j, m}\right\},
$$

for some $\boldsymbol{E}_{R} \in F_{p}$ and real numbers $c_{j, m}$ and $\tilde{c}_{j, m}$. The conclusion follows with

$$
\boldsymbol{E}=\boldsymbol{E}_{R}+\sum_{j=1}^{N}\left\{\sum_{\substack{\lambda_{j, m}-1 \in E_{j} \\ \frac{-2}{q}<\lambda_{j, m}<1}} c_{j, m} \boldsymbol{S}_{j, m}-\sum_{\substack{\tilde{\lambda}_{j, m}+1 \in E_{j} \\ \frac{-2}{q}<\tilde{c}_{j, m} \leq-1}} \tilde{c}_{j, m} \tilde{\boldsymbol{S}}_{j, m}\right\} .
$$

We easily come back to the ordinary Sobolev spaces since $P^{2, p}(\Omega) \subset W^{2, p}(\Omega)$. We actually keep the same decomposition because all singular functions in Theorem 4.12 do not belong to $W^{2, p}(\Omega)^{2}$. So the analogue of Theorem 4.12 with a regular part in $W^{2, p}(\Omega)^{2}$ holds:

Theorem 4.13. Let $p \geq 4 / 3$ and suppose that (3.14) holds. Then for each $\boldsymbol{J} \in$ $L^{p}(\Omega)^{2}$, there exist unique real numbers $c_{j, m}, \tilde{c}_{j, m}$ and a unique $\boldsymbol{E} \in X_{N}(\Omega)$ solution of the variational problem (2.6) such that

$$
\boldsymbol{E}-\sum_{j=1}^{N}\left\{\sum_{\substack{\lambda_{j, m}-1 \in E_{j} \\ \frac{-2}{q}<\lambda_{j, m}<1}} c_{j, m} \boldsymbol{S}_{j, m}-\sum_{\substack{\tilde{\lambda}_{j, m}+1 \in E_{j} \\ \frac{-2}{q}<\tilde{\lambda}_{j, m} \leq-1}} \tilde{\boldsymbol{c}}_{j, m} \tilde{\boldsymbol{S}}_{j, m}\right\} \in W^{2, p}(\Omega)^{2} .
$$

Remark 4.14. If $p=2$, the previous results is in accordance with [11, Theorem 3.4] again due to Remark 4.5. 
Remark 4.15. For $p \leq 2$, the above theorem implies that $\operatorname{div} \boldsymbol{E} \in W^{1, p}(\Omega)$ (thanks to Lemma 4.10), which is in accordance with Lemma 2.2. On the contrary if $p>2$, the above theorem (and again Lemma 4.10) restitutes the decomposition of $\operatorname{div} \boldsymbol{E}$ into a regular part and a singular part from Remark 2.3. Hence Theorem 4.13 respects the regularity of the divergence described in Section 2.

The previous theorem directly yields the next regularity results.

\section{Theorem 4.16.}

i) If $\omega_{j} \leq \frac{\pi}{3}$, for all $j=1, \cdots, N$, then for all $p \in[4 / 3,2]$, the unique solution $\boldsymbol{E} \in X_{N}(\Omega)$ of the variational problem (2.6) with $\boldsymbol{J} \in L^{p}(\Omega)^{2}$ belongs to $W^{2, p}(\Omega)^{2}$.

ii) If $\omega_{j} \in\left(\frac{\pi}{3}, \frac{2 \pi}{3}\right)$, for all $j=1, \cdots, N$, then for all $p \in\left[4 / 3, \frac{2}{3-\pi / \omega}\right]$, the unique solution $\boldsymbol{E} \in X_{N}(\Omega)$ of the variational problem (2.6) with $\boldsymbol{J} \in L^{p}(\Omega)^{2}$ belongs to $W^{2, p}(\Omega)^{2}$.

iii) If $\omega_{j} \geq \frac{2 \pi}{3}$, for some $j=1, \cdots, N$, then the unique solution $\boldsymbol{E} \in X_{N}(\Omega)$ of the variational problem (2.6) with $\boldsymbol{J} \in L^{p}(\Omega)^{2}$, for $p>4 / 3$, does not belong to $W^{2, p}(\Omega)^{2}$ in general.

Proof. The two first items are proved by showing that there are no $\lambda_{j, m}$ such that $\frac{-2}{q}<\lambda_{j, m}<1$ and no $\tilde{\lambda}_{j, m}$ such that $\frac{-2}{q}<\tilde{\lambda}_{j, m} \leq-1$. On the contrary, in the third case, there exists at least one $\lambda_{j, m}$ such that $\frac{-2}{q}<\lambda_{j, m}<1$.

\section{Appendix}

We here collect some trace results in the weighted Sobolev spaces $P^{2, p}(\Omega)^{2}$ and appropriate Green's formula used before.

We first recall a standard Green formula.

Theorem 5.1. For $\boldsymbol{E} \in W^{2, p}(\Omega)^{2}$ and $\boldsymbol{F} \in W^{2, q}(\Omega)^{2}$ with $\frac{1}{p}+\frac{1}{q}=1$, we have

$$
\begin{aligned}
\int_{\Omega}\{\Delta \boldsymbol{E} \cdot \boldsymbol{F}-\boldsymbol{E} \cdot \Delta \boldsymbol{F}\} d x d y= & \sum_{j=1}^{N} \int_{\Gamma_{j}}\{\operatorname{curl} \boldsymbol{E} \cdot \boldsymbol{F} \times \boldsymbol{n}-\operatorname{curl} \boldsymbol{F} \cdot \boldsymbol{E} \times \boldsymbol{n}\} d \sigma \\
& -\sum_{j=1}^{N} \int_{\Gamma_{j}}\{\operatorname{div} \boldsymbol{E} \cdot \boldsymbol{F} \cdot \boldsymbol{n}-\operatorname{div} \boldsymbol{F} \cdot \boldsymbol{E} \cdot \boldsymbol{n}\} d \sigma .
\end{aligned}
$$

Proof. Direct consequence of the standard Green formula using the writing $\Delta \boldsymbol{E}=$ $\nabla \operatorname{div} \boldsymbol{E}-\operatorname{curl} \operatorname{curl} \boldsymbol{E}$. 
Now we recall $[15,19]$ that for any $s>0, \tilde{W}^{s, p}\left(\Gamma_{j}\right)$ is the space of functions $u$ such that its extension by zero outside $\Gamma_{j}$ belongs to $W^{s, p}(\mathbb{R})\left(\Gamma_{j}\right.$ being identified with an interval of $\mathbb{R})$. The norm of $u$ in $\tilde{W}^{s, p}\left(\Gamma_{j}\right)$ is simply defined as the $W^{s, p}(\mathbb{R})$ norm of its extension.

Lemma 5.2. For every $\left\{\left(f_{j, 1}, f_{j, 2}, g_{j, 1}, g_{j, 2}\right)\right\}_{1 \leq j \leq N} \in Y_{p}:=\Pi_{j=1}^{N} \tilde{W}^{2-\frac{1}{p}, p}\left(\Gamma_{j}\right)^{2} \times$ $\tilde{W}^{1-\frac{1}{p}, p}\left(\Gamma_{j}\right)^{2}$, there exists $\boldsymbol{F} \in P^{2, p}(\Omega)^{2}$ such that

$$
\boldsymbol{F} \cdot \boldsymbol{n}=f_{j, 1}, \boldsymbol{F} \times \boldsymbol{n}=f_{j, 2}, \operatorname{curl} \boldsymbol{F}=g_{j, 1}, \operatorname{div} \boldsymbol{F}=g_{j, 2} \text { on } \Gamma_{j} .
$$

Moreover, there exists a constant $c$ such that

$$
\|\boldsymbol{F}\|_{P^{2, p}(\Omega)^{2}} \leq c\left\|\left\{\left(f_{j, 1}, f_{j, 2}, g_{j, 1}, g_{j, 2}\right)\right\}_{1 \leq j \leq N}\right\|_{Y_{p}} .
$$

Proof. For a fixed $G=\left\{\left(f_{j, 1}, f_{j, 2}, g_{j, 1}, g_{j, 2}\right)\right\}_{1 \leq j \leq N} \in Y_{p}$, we associate an element $\tilde{G}=\left\{\left(\tilde{f}_{j, 1}, \tilde{f}_{j, 2}, \tilde{g}_{j, 1}, \tilde{g}_{j, 2}\right)\right\}_{1 \leq j \leq N}$ in the same space defined by

$$
\begin{aligned}
& \tilde{f}_{j, 1}=f_{j, 1} \cdot n_{1}-f_{j, 2} \cdot n_{2} \text { on } \Gamma_{j} \\
& \tilde{f}_{j, 2}=f_{j, 1} \cdot n_{2}+f_{j, 2} \cdot n_{1} \text { on } \Gamma_{j} \\
& \tilde{g}_{j, 1}=g_{j, 2} \cdot n_{1}-g_{j, 1} \cdot n_{2}+\frac{\partial \tilde{f}_{j, 2}}{\partial t} \text { on } \Gamma_{j} \\
& \tilde{g}_{j, 2}=g_{j, 1} \cdot n_{1}+g_{j, 2} \cdot n_{2}-\frac{\partial \tilde{f}_{j, 1}}{\partial t} \text { on } \Gamma_{j},
\end{aligned}
$$

where $\left(n_{1}, n_{2}\right)$ is the unit outer normal vector along $\Gamma_{j}$. Obviously, we have

$$
\left\|\tilde{f}_{j, i}\right\|_{\tilde{W}^{2-\frac{1}{p}, p}\left(\Gamma_{j}\right)} \leq \sum_{l=1}^{2}\left\|f_{j, l}\right\|_{\tilde{W}^{2-\frac{1}{p}, p}\left(\Gamma_{j}\right)}, i=1,2,
$$

while

$$
\begin{aligned}
\left\|\tilde{g}_{j, i}\right\|_{\tilde{W}^{1-\frac{1}{p}, p}\left(\Gamma_{j}\right)} \leq & \sum_{l=1}^{2}\left\|g_{j, l}\right\|_{\tilde{W}^{1-\frac{1}{p}, p}\left(\Gamma_{j}\right)} \\
& +c \sum_{l=1}^{2}\left\|f_{j, l}\right\|_{\tilde{W}^{2-\frac{1}{p}, p}\left(\Gamma_{j}\right)}, i=1,2,
\end{aligned}
$$

since $\left\|\frac{\partial \tilde{f}_{j, i}}{\partial t}\right\|_{\tilde{W}^{1-\frac{1}{p}, p}\left(\Gamma_{j}\right)} \leq c\left\|\tilde{f}_{j, i}\right\|_{\tilde{W}^{2-\frac{1}{p}, p}\left(\Gamma_{j}\right)}$ by [15, Theorem 1.4.4.6].

On the other hand, thanks to [19, Theorems 1.31 and 1.35], we know that the mapping

$$
u \longmapsto\left\{\left(\gamma_{j} u, \gamma_{j} \frac{\partial u}{\partial n_{j}}\right)\right\}_{1 \leq j \leq N}
$$

maps continuously $P^{2, p}(\Omega)$ onto $Y_{p}$. Let us denote by $R$, its right continuous inverse and by $\|R\|$ the norm of $R$ from $Y_{p}$ to $P^{2, p}(\Omega)$. 
Applying this result to $\left\{\left(\tilde{f}_{j, i}, \tilde{g}_{j, i}\right)\right\}_{1 \leq j \leq N}, i=1,2$, we deduce the existence of two functions $F_{i}=R\left\{\left(\tilde{f}_{j, i}, \tilde{g}_{j, i}\right)\right\}_{1 \leq j \leq N} \in P^{2, p}(\Omega), i=1,2$ such that

$$
\gamma_{j} F_{i}=\tilde{f}_{j, i}, \gamma_{j} \frac{\partial F_{i}}{\partial n_{j}}=\tilde{g}_{j, i} \text { on } \Gamma_{j},
$$

and

$$
\left\|F_{i}\right\|_{P^{2, p}(\Omega)} \leq\|R\|\|\tilde{G}\|_{Y_{q}} .
$$

Now by construction of $\tilde{f}_{j, i}$ and $\tilde{g}_{j, i}$, we easily check that $\boldsymbol{F}=\left(F_{1}, F_{2}\right) \in P^{2, p}(\Omega)^{2}$ satisfies (5.1). Finally (5.5) with (5.3) and (5.4) leads to (5.2).

Before going on, we denote by $D\left(\Delta, L^{p}(\Omega)\right)=\left\{u \in L^{p}(\Omega): \Delta u \in L^{p}(\Omega)\right\}$, the maximal domain of the Laplace operator in $L^{p}(\Omega)$, which is a Banach space with the natural norm $\left(\|u\|_{0, p, \Omega}^{p}+\|\Delta u\|_{0, p, \Omega}^{p}\right)^{1 / p}[15,19]$.

Theorem 5.3. The mapping

$$
\boldsymbol{E} \longmapsto\{\boldsymbol{E} \cdot \boldsymbol{n}, \boldsymbol{E} \times \boldsymbol{n}, \operatorname{curl} \boldsymbol{E}, \operatorname{div} \boldsymbol{E}\}
$$

which is defined for $\boldsymbol{E} \in W^{2, p}(\Omega)^{2}$, has a unique continuous extension as an operator from $D\left(\Delta, L^{p}(\Omega)\right)^{2}$ into $\Pi_{j=1}^{N}\left(\tilde{W}^{1-\frac{1}{q}, q}\left(\Gamma_{j}\right)^{\prime}\right)^{2} \times\left(\tilde{W}^{2-\frac{1}{q}, q}\left(\Gamma_{j}\right)^{\prime}\right)^{2}$.

Proof. For $\boldsymbol{E} \in W^{2, p}(\Omega)^{2}$ and $\boldsymbol{F} \in P^{2, q}(\Omega)^{2}$ with $\frac{1}{p}+\frac{1}{q}=1$, we have from Theorem 5.1

$$
\begin{aligned}
\int_{\Omega}\{\Delta \boldsymbol{E} \cdot \boldsymbol{F}-\boldsymbol{E} \cdot \Delta \boldsymbol{F}\} d x d y= & \sum_{j=1}^{N} \int_{\Gamma_{j}}\{\operatorname{curl} \boldsymbol{E} \cdot \boldsymbol{F} \times \boldsymbol{n}-\operatorname{curl} \boldsymbol{F} \cdot \boldsymbol{E} \times \boldsymbol{n}\} d \sigma \\
& -\sum_{j=1}^{N} \int_{\Gamma_{j}}\{\operatorname{div} \boldsymbol{E} \cdot \boldsymbol{F} \cdot \boldsymbol{n}-\operatorname{div} \boldsymbol{F} \cdot \boldsymbol{E} \cdot \boldsymbol{n}\} d \sigma .
\end{aligned}
$$

It follows from Hölder's inequality that

$$
\begin{aligned}
& \sum_{j=1}^{N} \int_{\Gamma_{j}}\{\operatorname{curl} \boldsymbol{E} \cdot \boldsymbol{F} \times \boldsymbol{n}-\operatorname{curl} \boldsymbol{F} \cdot \boldsymbol{E} \times \boldsymbol{n}\} d \sigma \\
& -\sum_{j=1}^{N} \int_{\Gamma_{j}}\{\operatorname{div} \boldsymbol{E} \cdot \boldsymbol{F} \cdot \boldsymbol{n}-\operatorname{div} \boldsymbol{F} \cdot \boldsymbol{E} \cdot \boldsymbol{n}\} d \sigma \leq C\|\boldsymbol{E}\|_{D\left(\Delta, L^{p}(\Omega)\right)^{2}}\|\boldsymbol{F}\|_{P^{2, q}(\Omega)^{2} .}
\end{aligned}
$$

Let us now fix $\boldsymbol{E} \in W^{2, p}(\Omega)^{2}$ and define the continuous linear form $l$ on $Y_{q}$ by

$$
\begin{aligned}
l\left(\left\{\left(f_{j, 1}, f_{j, 2}, g_{j, 1}, g_{j, 2}\right)\right\}_{1 \leq j \leq N}\right)= & \sum_{j=1}^{N} \int_{\Gamma_{j}}\left\{\operatorname{curl} \boldsymbol{E} \cdot f_{j, 2}-g_{j, 1} \cdot \boldsymbol{E} \times \boldsymbol{n}\right\} d \sigma \\
& -\sum_{j=1}^{N} \int_{\Gamma_{j}}\left\{\operatorname{div} \boldsymbol{E} \cdot f_{j, 1}-g_{j, 2} \cdot \boldsymbol{E} \cdot \boldsymbol{n}\right\} d \sigma .
\end{aligned}
$$


In order to estimate the norm of $l$ in $Y_{q}^{\prime}$, we take advantage of Lemma 5.2. Thus for a fixed $G=\left\{\left(f_{j, 1}, f_{j, 2}, g_{j, 1}, g_{j, 2}\right)\right\} \in Y_{q}$, there exists $\boldsymbol{F} \in P^{2, q}(\Omega)^{2}$ satisfying (5.1) and (5.2). Going back to (5.8) and taking into account (5.7) we obtain

$$
|l(G)| \leq C\|\boldsymbol{E}\|_{D\left(\Delta, L^{p}(\Omega)\right)^{2}}\|G\|_{Y_{q}} .
$$

This means that the mapping (5.6) is continuous from $W^{2, p}(\Omega)^{2}$ equipped with the norm of $D\left(\Delta, L^{p}(\Omega)\right)^{2}$ into $Y_{q}^{\prime}$. Therefore, from the density of $W^{2, p}(\Omega)$ into $D\left(\Delta, L^{p}(\Omega)\right.$ ) (see Lemma 1.36 of [19]), we deduce that this mapping can be extended in a unique way to $D\left(\Delta, L^{p}(\Omega)\right)^{2}$.

Corollary 5.4. For all $\boldsymbol{E} \in D\left(\Delta, L^{p}(\Omega)\right)^{2}$ and $\boldsymbol{F} \in P^{2, q}(\Omega)^{2}$ with $\frac{1}{p}+\frac{1}{q}=1$, we have

$$
\begin{aligned}
\int_{\Omega}\{\Delta \boldsymbol{E} \cdot \boldsymbol{F}-\boldsymbol{E} \cdot \Delta \boldsymbol{F}\}= & \sum_{j=1}^{N}\left(\langle\operatorname{curl} \boldsymbol{E} ; \boldsymbol{F} \times \boldsymbol{n}\rangle_{\Gamma_{j}}-\langle\boldsymbol{E} \times \boldsymbol{n} ; \operatorname{curl} \boldsymbol{F}\rangle_{\Gamma_{j}}\right) \\
& -\sum_{j=1}^{N}\left(\langle\operatorname{div} \boldsymbol{E} ; \boldsymbol{F} \cdot \boldsymbol{n}\rangle_{\Gamma_{j}}-\langle\boldsymbol{E} \cdot \boldsymbol{n} ; \operatorname{div} \boldsymbol{F}\rangle_{\Gamma_{j}}\right),
\end{aligned}
$$

where $\langle\cdot ; \cdot\rangle_{\Gamma_{j}}$ is the duality pairing between $\left(\tilde{W}^{1-\frac{1}{q}, q}\left(\Gamma_{j}\right)^{\prime}\right)^{2}$ and $\tilde{W}^{1-\frac{1}{q}, q}\left(\Gamma_{j}\right)^{2}$, or between $\left(\tilde{W}^{2-\frac{1}{q}, q}\left(\Gamma_{j}\right)^{\prime}\right)^{2}$ and $\tilde{W}^{2-\frac{1}{q}, q}\left(\Gamma_{j}\right)^{2}$.

\section{References}

[1] S. Agmon, A. Douglis and L. Nirenberg, Estimates near the boundary for solutions of elliptic partial differential equations satisfying general conditions II, Comm. Pure Appl. Math. 17 (1964), 35-92.

[2] A. Aibeche, W. Chikouche and S. Nicaise, $l^{p}$ regularity of transmission problems in dihedral domains, Boll. Unione Mat. Ital. Sez. B Artic. Ric. Mat. (8) 10-B (2007), 663-660.

[3] C. Amrouche, C. Bernardi, M. Dauge and V. Girault, Vector potentials in threedimensional nonsmooth domains, Math. Methods Appl. Sci. 21 (1998), 823-864.

[4] T. APEL and S. NICAISE, The finite element method with anisotropic mesh grading for elliptic problems in domains with corners and edges, Math. Methods Appl. Sci. 21 (1998), 519-549.

[5] F. Assous and P. Ciarlet, Une caractérisation de l'orthogonal de $\Delta\left(H^{2}(\Omega) \cap H_{0}^{1}(\Omega)\right)$ dans $L^{2}(\Omega)$, C. R. Acad. Sc. Paris, Série I 325 (1997), 605-610.

[6] F. Assous, P. Ciarlet JR. and E. SonnendrüCKER, Résolution of the Maxwell equations in a domain with reentrant corners, RAIRO Modél. Math. Anal. Numér. 32 (1998), 359-389.

[7] M. Birman and M. Z. SOlOMYAK, $L^{2}$ theory of the Maxwell operator in arbitrary domains, Russian Math. Surveys 42 (1987), 75-96.

[8] M. S. BIRMAN and M. Z. SOLOMYAK, On the main singularities of the electric component of the electro-magnetic field in regions with screens, St. Petersburg Math. J. 5 (1993), 125139. 
[9] P. ClÉment and P. GRISVARD, Somme d'opérateurs et régularité $L^{p}$ dans les problèmes aux limites, C. R. Acad. Sci. Paris Série I, 314 (1992), 821-824.

[10] M. COSTABEL A remark on the regularity of solutions of Maxwell's equations on Lipschitz domains, Math. Methods Appl. Sci. 12 (1990), 365-368.

[11] M. Costabel and M. DaUge, Singularities of electromagnetic fields in polyhedral domains Arch. Ration. Mech. Anal. 151 (2000), 221-276.

[12] G. DA PRATO and P. GRISVARD, Somme d'opérateurs linéaires et équations différentielles opérationnelles, J. Math. Pures Appl., Ser. IX, 54 (1975), 305-387.

[13] G. Dore and A. VenNI, On the closedness of the sum of two closed operators, Math. Z. 196 (1987), 189-201.

[14] V. Giraultand and P.-A. Raviart, "Finite Element Methods for Navier-Stokes Equations, Theory and Algorithms", Vol. 5, Springer Series in Computational Mathematics, Springer, Berlin, 1986.

[15] P. GRISVARD, "Elliptic Problems in Nonsmooth Domains", Vol. 24, Monographs and Studies in Mathematics, Pitman, Boston-London-Melbourne, 1985.

[16] M. D. Gunzburger, A. J. Meir and J. S. Peterson, On the existence, uniqueness, and finite element approximation of solutions of the equations of stationary, incompressible magnetohydrodynamics, Math. Comp. 56 (1991), 523-563.

[17] V. G. MAZ'YA and B. A. PLAMENEVSKII, Estimates in $L^{p}$ and in Hölder classes and the Miranda-Agmon maximum principle for solutions of elliptic boundary value problems in domains with singular points on the boundary, Amer. Math. Soc. Transl. Ser. 2, 123 (1984), $1-56$.

[18] M. Moussaoui Espace H(curl, div) dans un polygone plan, C. R. Acad. Sc. Paris, Série I, 322 (1996), 225-229.

[19] S. NiCAISE, " Polygonal Interface Problems", Vol. 39, Methoden und Verfahren der mathematischen Physik, Peter Lang GmbH, Europäischer Verlag der Wissenschaften, Frankfurt/M., 1993.

[20] S. NICAISE, Edge elements on anisotropic meshes and approximation of the Maxwell equations, SIAM J. Numer. Anal. 39 (2001), 784-816(electronic).

[21] C. Weber A local compactness theorem for Maxwell's equations, Math. Methods Appl. Sci. 2 (1980), 12-25.

Université de Jijel

Laboratoire de Mathematiques pures

et Appliquées

Faculté des Sciences

B.P. 98, Ouled Aissa

18000 Jijel, Algeria

chikouche@mail.univ-jijel.dz

Université de Valenciennes

et du Hainaut Cambrésis

LAMAV, FR CNRS 2956

and

Institut des Sciences

et Techniques de Valenciennes

F-59313 - Valenciennes Cedex 9, France

serge.nicaise@univ-valenciennes.fr 